\title{
Numerical methods for the Stokes and Navier-Stokes equations driven by threshold slip boundary conditions
}

\author{
Jules DJOKO* Jonas $\mathrm{KOKO}^{\dagger}$
}

January 29, 2016

\begin{abstract}
In this article, we discuss the numerical solution of the Stokes and Navier-Stokes equations completed by nonlinear slip boundary conditions of friction type in two and three dimensions. To solve the Stokes system, we first reduce the related variational inequality into a saddle point-point problem for a well chosen augmented Lagrangian. To solve this saddle point problem we suggest an alternating direction method of multiplier together with finite element approximations. The solution of the Navier Stokes system combines finite element approximations, time discretization by operator splitting and augmented Lagrangian method. Numerical experiment results for two and three dimensional flow confirm the interest of these approaches.
\end{abstract}

Keywords. nonlinear slip boundary condition, Stokes equations, NavierStokes equations, variational inequality, augmented Lagrangian, alternating direction method of multipliers, Marchuk-Yanenko's scheme, 3d-simulations.

\section{Introduction}

Numerical solutions for variational inequalities have been examined by many researchers, see for example, [22, 27, 28, 36, 47]. Roughly speaking, there are two main approaches to solve variational inequalities: the direct treatment of the inequality by making use of minimization techniques, and the transformation of the variational inequality into a variational equation by regularization or by introducing a "multiplier". It should be acknowledged

\footnotetext{
*Departement of Mathematics and Applied Mathematics, University of Pretoria, Private bag X20, Hatfield 0028, Pretoria, South Africa; corresponding author,Email: jules.djokokamdem@up.ac.za

${ }^{\dagger}$ Clermont Université, LIMOS, UMR 6158 CNRS - Université Blaise Pascal, BP 10448, F-63000 Clermont-Ferrand, France; Email: jonas.koko@univ-bpclermont.fr
} 
that earlier works on numerical analysis of variational inequalities were concerned with applications to solid mechanics (see [20, 22, 27, 28, 36, 47] just to cite a few). In $[28,36,47]$, the authors have considered static or quasistatic contact problems in the theory of elasticity, viscoelastic, viscoplastic, damage that can be formulated as variational inequalities. A comprehensive analysis of these problems are discussed in a view to unify the physical problems with mathematical modeling of the phenomena and their numerical implementation. In [27], the focus is on modeling, mathematical and numerical analysis of plastic deformations, while in [20,22], the authors have discussed the solution strategies (including existence theory and convergence of numerical schemes) in a very general framework of variational inequalities, with references to problems in contact, plasticity.

Recently, due to some advances in modeling it has been shown that some natural flows may have nonlinear frictional slip at the interface separating the fluid and the solid, giving rise to variational inequality (see $[11,15,14$, $13,16,43,44,42,45])$. The numerical investigation of these later problems have benefited a lot from the existing knowledge of general ways of treating variational inequalities and some works worth to be mentioned here include $[2,40,39,30,7,18]$.

It should be noted that one of the first breakthrough for a systematic mathematical analysis of problems formulated as variational inequalities was due to G. Duvaut and J.L. Lions [8].

The main goal of our work is to discuss the numerical solution of some fluid flows driven by nonlinear slip boundary conditions of friction type. These include the Stokes, Navier Stokes in two and three dimensions. The point of departure of this study is the work of J.K. Djoko and M. Mbehou [7], where the resulting formulation had been solved by making use of the "Lagrange multiplier" and application of Uzawa's algorithm. In this work, we solve the problem associated with the Stokes equations by exploiting the minimization structure of the variational formulation, and apply to it an alternating direction method reminiscent to those used in $[6,22,20,18,38$, 37]. Next, we solve the stationary Navier Stokes equations in two steps. Firstly, we associate with the stationary problem a time dependent problem in which only the long time behavior is considered. The time dependent problem is solved using an operator splitting scheme.

The augmented Lagrangian approach has been used recently in $[18,38$, 37 ] to overcome the difficulty encountered in the variational formulation of Stokes flow driven by degree one homogeneous convex, lower semi-continuous and non-differentiable boundary conditions (Tresca friction law). In fact the method used by M. K. Gdoura, J. Koko and T. Sassi [18] combined a domain decomposition approach together with augmented Lagrangian formulation and the Uzawa block relaxation technique. In [38, 37], the augmented Lagrangian approach is associated with three Lagrange multipliers, each having a specific role to play. The algorithms presented here are inspired by the 
works of $[20,18,38,37]$, and the mathematical merits of these schemes are thoroughly discussed in $[20,23,36,25]$. It should be mentioned though that besides the common problems encountered when one solves Stokes or Navier Stokes equations, one of the major challenges in this work is the presence in the variational formulation of the non-classical dissipation term $j(\boldsymbol{u}, \boldsymbol{v})$ (see (2.8)) which brings some interesting variations/ twist in our analysis. Hence, the algorithms presented are in a way extensions (re-adaptation) of existing ones, and their numerical implementation are more involved. This article also discusses for the first time the implementation of such phenomena in three dimensional space.

The remainder of this work is divided as follows. In Section 2, we present the Stokes and Navier-Stokes equations completed by nonlinear slip boundary conditions of friction type. Section 3 deals with the alternating direction method of multiplier (ADDM) for the Stokes equations, and Navier Stokes equations being treated in Section 4, by considering an operator splitting/ADDM algorithm where only the long term effect of an associated time evolution problem is considered. The space discretization with stabilised $P_{1}-P_{1}$ is outlined in Section 5 and Section 6 deals with numerical simulations.

\section{Preliminaries and Weak formulations}

We consider the flow of a viscous incompressible fluid confined in $\Omega$, modeled by the Stokes system;

$$
\begin{aligned}
\alpha \boldsymbol{u}-2 \nu \operatorname{div} \boldsymbol{D}(\boldsymbol{u})+\nabla p & =\boldsymbol{f} & & \text { in } \Omega, \\
\operatorname{div} \boldsymbol{u} & =0 & & \text { in } \Omega,
\end{aligned}
$$

where the flow region $\Omega$, is a bounded domain in $\mathbb{R}^{d}$ with $d=2,3$. The motion of the incompressible fluid is described by the velocity $\boldsymbol{u}(\boldsymbol{x})$ and pressure $p(\boldsymbol{x})$. In (2.1) $\boldsymbol{f}$ is the external body force per unit volume depending on $\boldsymbol{x}$, and $\nu>0$ is the positive parameter representing the kinetic viscosity, while $\alpha \geq 0$ is a positive constant. If $\alpha=0,(2.1)-(2.2)$ is the classical Stokes problem. Equations (2.1) and (2.2) are supplemented by nonlinear slip boundary of friction type, which is the main modeling assumption in this work. It should be pointed out that such boundary conditions have already been considered in $[43,44]$. Hence we will just state the mathematical equations governing this phenomenon as the physical merits of such model have been discussed elsewhere (see particularly [43]). So, we assume that the boundary of $\Omega$, say, $\partial \Omega$ is made of two components $S$ and $\Gamma$, and it is required that $\partial \Omega=\overline{S \cup \Gamma}$, with $S \cap \Gamma=\emptyset$. Next, we consider the homogeneous Dirichlet condition on $\Gamma$, that is

$$
\boldsymbol{u}=\mathbf{0} \quad \text { on } \Gamma \text {. }
$$


On the boundary $S$, we assume that the velocity can be decomposed as follows

$$
\boldsymbol{u}=\boldsymbol{u}_{\boldsymbol{\tau}}+u_{n} \boldsymbol{n}
$$

with $u_{n}=\boldsymbol{u} \cdot \boldsymbol{n}$, and $\boldsymbol{n}$ stands for the outward, normal unit vector on $S$. we first assume the impermeability condition, that is

$$
\boldsymbol{u} \cdot \boldsymbol{n}=0 \text { on } S .
$$

In addition to (2.4), we also impose on $S$, a nonlinear slip boundary condition of friction type which is describe next. Considering the Cauchy-stress tensor $\boldsymbol{T}=-p \boldsymbol{I}+2 \nu \boldsymbol{D}(\boldsymbol{u})$, with the symmetric part of the velocity gradient $\boldsymbol{D}(\boldsymbol{u})=\frac{1}{2}\left[\nabla \boldsymbol{u}+(\nabla \boldsymbol{u})^{T}\right]$, we assume that the traction force $\boldsymbol{T} \boldsymbol{n}$ on $S$ is decomposed as follows

$$
\boldsymbol{T n}=(\boldsymbol{T n} \cdot \boldsymbol{n}) \boldsymbol{n}+(\boldsymbol{T n})_{\tau} .
$$

On $S$, the main modeling assumption is formulated as follows

$$
\left.\begin{array}{l}
\left|(\boldsymbol{T} \boldsymbol{n})_{\boldsymbol{\tau}}\right|<g \Rightarrow \boldsymbol{u}_{\boldsymbol{\tau}}=\mathbf{0} \\
\left|(\boldsymbol{T} \boldsymbol{n})_{\boldsymbol{\tau}}\right| \geq g \Rightarrow \boldsymbol{u}_{\boldsymbol{\tau}} \neq \mathbf{0} \text { and }(\boldsymbol{T} \boldsymbol{n})_{\boldsymbol{\tau}}=-\left(g+\kappa\left|\boldsymbol{u}_{\boldsymbol{\tau}}\right|\right) \frac{\boldsymbol{u}_{\boldsymbol{\tau}}}{\left|\boldsymbol{u}_{\boldsymbol{\tau}}\right|}
\end{array}\right\} \text { on } S,
$$

where $\kappa$ is a positive value, standing for the friction coefficient, the vector length is denoted by $|\cdot|, g: S \rightarrow(0, \infty)$ is the slip threshold or barrier function. We will refer to the boundary-value problem (2.1) - (2.5) as problem (S). We will also investigate the Navier-Stokes system of equations, where (2.1) is replaced by

$$
\alpha \boldsymbol{u}-2 \nu \operatorname{div} \boldsymbol{D}(\boldsymbol{u})+(\boldsymbol{u} \cdot \nabla) \boldsymbol{u}+\nabla p=\boldsymbol{f} \quad \text { in } \Omega,
$$

and (2.2), (2.3), (2.4) and (2.5) are unchanged. Here,

$$
(\boldsymbol{v} \cdot \nabla) \boldsymbol{v}=\sum_{i=1}^{d} v_{i} \frac{\partial \boldsymbol{v}}{\partial x_{i}}
$$

is the convection term. We will refer to boundary-value problem (2.2) (2.6), as problem (NS). If $\alpha$ is greater than zero in (2.1) and (2.6), then it is the reciprocal of a time-step, its presence indicating that our methodology can handle time dependant problems. It should quickly be mentioned that (2.5) is equivalent to (see [8])

$$
(\boldsymbol{T n})_{\boldsymbol{\tau}} \cdot \boldsymbol{u}_{\boldsymbol{\tau}}+\left(g+\kappa\left|\boldsymbol{u}_{\boldsymbol{\tau}}\right|\right)\left|\boldsymbol{u}_{\boldsymbol{\tau}}\right|=0 \text { on } S,
$$

which is re-written with the use of sub-differential as

$$
-(\boldsymbol{T n})_{\boldsymbol{\tau}} \in\left(g+\kappa\left|\boldsymbol{u}_{\boldsymbol{\tau}}\right|\right) \partial\left|\boldsymbol{u}_{\boldsymbol{\tau}}\right| \text { on } S,
$$


where $\partial|\cdot|$ is the sub-differential of the real valued function $|\cdot|$ with $|\boldsymbol{w}|^{2}=\boldsymbol{w} \cdot \boldsymbol{w}$. At this juncture, it should be noted that nonlinear slip boundary conditions of friction type were introduced by Fujita in [11], where he studied some hydrodynamics problems, such as the blood flow in a vein of an arterial sclerosis patient and the avalanche of water and rocks. Subsequently, many studies have focused on the properties of the solution of the resulting boundary value problem, for example, existence, uniqueness, regularity, and continuous dependence on data, for Stokes, Navier-Stokes and Brinkman-Forchheimer equations under such boundary conditions. Details can be found in $[43,11,12,3,44,1,45,46,14,13,15,16,17]$ among others. To derive the weak formulations associated with problems (S), (NS), we recall that standard definitions and notations applies (see [8]). We denote by $H^{1}(\Omega)$ and $\|\cdot\|_{1}$ the standard Sobolev space and its norm, respectively. $(a, b)$, is the real $L^{2}$ inner product of $a$ and $b$, while $\|\cdot\|$ is the norm in $L^{2}$. Boldface letters will be related to vector spaces, for instance $L^{2}(\Omega)$ stands for $L^{2}(\Omega) \times L^{2}(\Omega)$, etc.... We first introduced the classical spaces

$$
\begin{aligned}
\boldsymbol{V} & =\left\{\boldsymbol{v} \in \boldsymbol{H}^{1}(\Omega):\left.\boldsymbol{v}\right|_{\Gamma}=\mathbf{0},\left.v_{n}\right|_{S}=0\right\} \\
M & =L_{0}^{2}=\left\{p \in L^{2}(\Omega):(p, 1)=0\right\} \\
\boldsymbol{V}_{\operatorname{div}} & =\left\{\boldsymbol{v} \in \boldsymbol{H}^{1}(\Omega):\left.\operatorname{div} \boldsymbol{v}\right|_{\Omega}=0,\left.\quad \boldsymbol{v}\right|_{\Gamma}=\mathbf{0},\left.v_{n}\right|_{S}=0\right\} .
\end{aligned}
$$

We let

$$
\begin{aligned}
& a: \quad H^{1}(\Omega)^{2} \quad \longrightarrow \mathbb{R} \\
& (\boldsymbol{v}, \boldsymbol{u}) \quad \longrightarrow a(\boldsymbol{v}, \boldsymbol{u})=\alpha(\boldsymbol{u}, \boldsymbol{v})+2 \nu(\boldsymbol{D}(\boldsymbol{v}), \boldsymbol{D}(\boldsymbol{u})), \\
& d: \quad \boldsymbol{H}^{1}(\Omega)^{3} \quad \longrightarrow \mathbb{R} \\
& (\boldsymbol{u}, \boldsymbol{v}, \boldsymbol{w}) \quad \longrightarrow d(\boldsymbol{u}, \boldsymbol{v}, \boldsymbol{w})=((\boldsymbol{u} \cdot \nabla) \boldsymbol{v}, \boldsymbol{w}), \\
& b: \quad \boldsymbol{H}^{1}(\Omega) \times M \quad \longrightarrow \mathbb{R} \\
& (\boldsymbol{v}, q) \quad \longrightarrow b(\boldsymbol{v}, q):=-(\operatorname{div} \boldsymbol{v}, q), \\
& j: \quad \boldsymbol{H}^{1}(\Omega)^{2} \quad \longrightarrow \mathbb{R} \\
& (\boldsymbol{v}, \boldsymbol{w}) \quad \longrightarrow j(\boldsymbol{v})=\left(g+\kappa\left|\boldsymbol{v}_{\boldsymbol{\tau}}\right|,\left|\boldsymbol{w}_{\boldsymbol{\tau}}\right|\right)_{S}, \\
& \ell: \quad \boldsymbol{H}^{1}(\Omega) \quad \longrightarrow \mathbb{R} \\
& \boldsymbol{v} \quad \longrightarrow \quad \ell(\boldsymbol{v})=(\boldsymbol{f}, \boldsymbol{v}) \text {. }
\end{aligned}
$$

Throughout this work, we will used the following facts

$$
\begin{aligned}
& 2(\boldsymbol{a}-\boldsymbol{b}, \boldsymbol{a})=\|\boldsymbol{a}\|^{2}-\|\boldsymbol{b}\|^{2}+\|\boldsymbol{a}-\boldsymbol{b}\|^{2}, \text { for all } \boldsymbol{a}, \boldsymbol{b} \in \boldsymbol{L}^{2}(\Omega) . \\
& a b \leq \frac{\epsilon}{p} a^{p}+\frac{1}{q \epsilon^{q / p}} b^{q} \text { for all } a, b, \epsilon>0, \text { with } \frac{1}{p}+\frac{1}{q}=1 .
\end{aligned}
$$

It should be noted that (see [19]):

(a) The bilinear and linear forms $a(\cdot, \cdot), b(\cdot, \cdot)$ and $\ell$ are continuous on $\boldsymbol{V}$;

(b) There exists a constant $c_{\Omega}>0$ depending on the domain $\Omega$ such that

$$
a(\boldsymbol{v}, \boldsymbol{v}) \geq 2 \nu c_{\Omega}\|\boldsymbol{v}\|_{1}^{2} \text { for all } \boldsymbol{v} \in \boldsymbol{V} .
$$


(c) There exists a constant $\beta_{\Omega}>0$ depending only on $\Omega$ such that

$$
\beta_{\Omega}\|q\| \leq \sup _{\boldsymbol{v} \in \boldsymbol{V}} \frac{b(\boldsymbol{v}, q)}{\|\boldsymbol{v}\|_{1}} \text { for all } \boldsymbol{v} \in \boldsymbol{V} .
$$

The weak formulation associated with $(2.1)-(2.5)$ is as follows:

$$
\left\{\begin{array}{l}
\operatorname{problem}(\mathbf{S}): \\
\text { Find }(\boldsymbol{u}, p) \in \boldsymbol{V} \times M \text { such that for all }(\boldsymbol{v}, q) \in \boldsymbol{V} \times M \\
a(\boldsymbol{u}, \boldsymbol{v}-\boldsymbol{u})+b(\boldsymbol{v}-\boldsymbol{u}, p)+j(\boldsymbol{u}, \boldsymbol{v})-j(\boldsymbol{u}, \boldsymbol{u}) \geq \ell(\boldsymbol{v}-\boldsymbol{u}) \\
b(\boldsymbol{v}, q)=0
\end{array}\right.
$$

The variational problem (2.13) is equivalent to

$$
\left\{\begin{array}{l}
\text { Find } \boldsymbol{u} \in \boldsymbol{V}_{\mathrm{div}} \text { such that } \\
a(\boldsymbol{u}, \boldsymbol{v}-\boldsymbol{u})+j(\boldsymbol{u}, \boldsymbol{v})-j(\boldsymbol{u}, \boldsymbol{u}) \geq \ell(\boldsymbol{v}-\boldsymbol{u}) \text { for all } \boldsymbol{w} \in \boldsymbol{V}_{\mathrm{div}}
\end{array}\right.
$$

Finally, observing that the frictional functional $j(\boldsymbol{v}, \boldsymbol{v})$ is convex, then $(2.12)$ is equivalent to the following minimization problem.

$$
\left\{\begin{array}{l}
\text { Find } \boldsymbol{u} \in \boldsymbol{V}_{\mathrm{div}} \text { such that for all } \boldsymbol{v} \in \boldsymbol{V} \\
J_{\boldsymbol{S}}(\boldsymbol{u}) \leq J_{\boldsymbol{s}}(\boldsymbol{v}) \\
J_{\boldsymbol{s}}(\boldsymbol{v})=\frac{1}{2} a(\boldsymbol{v}, \boldsymbol{v})-\ell(\boldsymbol{v})+j(\boldsymbol{v}, \boldsymbol{v})
\end{array}\right.
$$

We first claim that

\section{Theorem 2.1. ([7])}

(a) For all $\boldsymbol{v} \in \boldsymbol{H}^{1}(\Omega), j(\boldsymbol{v}, \boldsymbol{v})$ is convex and nonnegative continuous on $\boldsymbol{H}^{1}(\Omega)$.

(b) For all $\boldsymbol{v}_{1}, \boldsymbol{v}_{2}, \boldsymbol{w}_{1}, \boldsymbol{w}_{2} \in \boldsymbol{H}^{1}(\Omega)$, there exists $C_{0}$ depending on the domain $\Omega$ such that

$j\left(\boldsymbol{v}_{1}, \boldsymbol{w}_{2}\right)-j\left(\boldsymbol{v}_{1}, \boldsymbol{w}_{1}\right)+j\left(\boldsymbol{v}_{2}, \boldsymbol{w}_{1}\right)-j\left(\boldsymbol{v}_{2}, \boldsymbol{w}_{2}\right) \leq C_{0} k\left\|\boldsymbol{v}_{1}-\boldsymbol{v}_{2}\right\|_{1}\left\|\boldsymbol{w}_{1}-\boldsymbol{w}_{2}\right\|_{1}$.

(c) If $\kappa, \nu$ are given in such a way that

$$
0<\frac{C_{0} \kappa}{2 \nu}<1
$$

then the mixed variational problem (2.13) admits a unique solution $(\boldsymbol{u}, p) \in \boldsymbol{V} \times M$, which satisfies the following bound

$$
\|\boldsymbol{u}\|_{1}+\|p\| \leq C\left(\|\boldsymbol{f}\|+\|g\|_{L^{2}(S)}\right) .
$$


We recall the following result that will be used next.

Lemma 2.1. Let $V$ be a real reflexive Banach space, and $V^{*}$ be its dual space, and $K$ be a non-empty closed convex subset of $V$.

Let $J_{0}: V \longrightarrow \mathbb{R}$, be a convex functional, and $G$-differentiable on $V$, and $J_{1}: V \longrightarrow \overline{\mathbb{R}}$ be a proper i.s.c convex functional, and let $J=J_{0}+J_{1}$. Then the solution (if exists) of the minimization: Find $u \in K$ such that

$$
J(u) \leq J(v), \text { for all } v \in K,
$$

is characterized by

$$
\left\langle J_{0}^{\prime}(u), v-u\right\rangle+J_{1}(v)-J_{1}(u) \geq 0 \text { for all } v \in K .
$$

Turning to Navier-Stokes system, the weak formulation associated with $(2.2)-(2.6)$ reads

$$
\left\{\begin{array}{l}
\operatorname{problem}(\mathbf{N S}) \\
\text { Find }(\boldsymbol{u}, p) \in \boldsymbol{V} \times M \text { such that for all }(\boldsymbol{v}, q) \in \boldsymbol{V} \times M \\
a(\boldsymbol{u}, \boldsymbol{v}-\boldsymbol{u})+d(\boldsymbol{u}, \boldsymbol{u}, \boldsymbol{v}-\boldsymbol{u})+b(\boldsymbol{v}-\boldsymbol{u}, p) \\
+j(\boldsymbol{u}, \boldsymbol{v})-j(\boldsymbol{u}, \boldsymbol{u}) \geq \ell(\boldsymbol{v}-\boldsymbol{u}) \\
b(\boldsymbol{u}, q)=0
\end{array}\right.
$$

We also recall the following properties [48];

$$
\begin{aligned}
d(\boldsymbol{u}, \boldsymbol{v}, \boldsymbol{w}) & \leq C_{1}\|\nabla \boldsymbol{u} \mid\| \nabla \boldsymbol{v}\|\| \nabla \boldsymbol{w} \| \text { for all }(\boldsymbol{u}, \boldsymbol{v}, \boldsymbol{w}) \in \boldsymbol{V}^{3} \\
d(\boldsymbol{u}, \boldsymbol{v}, \boldsymbol{v}) & =0 \text { for all }(\boldsymbol{u}, \boldsymbol{v}, \boldsymbol{w}) \in \boldsymbol{V}_{\mathrm{div}} \times \boldsymbol{V} \times \boldsymbol{V}
\end{aligned}
$$

The solvability of problem (NS) is obtained by combining; Galerkin, fixed point approach and compactness method. In fact we claim that

Theorem 2.2. For any data $(\boldsymbol{f}, g) \in \boldsymbol{L}^{2}(\Omega) \times L^{2}(S)$, problem (NS) admits at least a solution $(\boldsymbol{u}, p) \in \boldsymbol{V} \times M$. Moreover assuming that there exists a positive constant $C_{*}$ such that if the data $(\boldsymbol{f}, g) \in \boldsymbol{L}^{2}(\Omega) \times L^{2}(S)$, $\nu$ and $\kappa$ satisfy

$$
\nu<C_{*}\left(\kappa+\|\boldsymbol{f}\|+\|g\|_{S}\right),
$$

then the solution is unique.

The proof of existence of solutions is obtained by implementing the fixed point approach due to Brouwer. It is done in several steps.

Step 1. We recall that $\boldsymbol{V}_{\text {div }}$ is a separable Hilbert space. Hence there exist $\left\{\boldsymbol{\phi}_{i}\right\}_{i=1}^{\infty}$ an orthonormal basis of $\boldsymbol{V}_{\text {div }}$. Let

$$
\boldsymbol{W}_{n}=\left\{\phi_{1}, \phi_{2}, \ldots, \boldsymbol{\phi}_{n}\right\}
$$


Then one considers the following problem;

$$
\left\{\begin{array}{l}
\text { Find } \boldsymbol{u}_{n} \in \boldsymbol{W}_{n} \text { such that for all } \boldsymbol{v} \in \boldsymbol{W}_{n}, \\
a\left(\boldsymbol{u}_{n}, \boldsymbol{v}-\boldsymbol{u}_{n}\right)+d\left(\boldsymbol{u}_{n}, \boldsymbol{u}_{n}, \boldsymbol{v}-\boldsymbol{u}_{n}\right)+j\left(\boldsymbol{u}_{n}, \boldsymbol{v}\right)-j\left(\boldsymbol{u}_{n}, \boldsymbol{u}_{n}\right) \\
\geq \ell\left(\boldsymbol{v}-\boldsymbol{u}_{n}\right) .
\end{array}\right.
$$

Secondly, for every $\widetilde{\boldsymbol{u}}_{n} \in \boldsymbol{W}_{n}$, consider the linearized problem

$$
\left\{\begin{array}{l}
\text { Find } \boldsymbol{u}_{n} \in \boldsymbol{W}_{n} \text { such that for all } \boldsymbol{v} \in \boldsymbol{W}_{n} \\
a\left(\boldsymbol{u}_{n}, \boldsymbol{v}-\boldsymbol{u}_{n}\right)+j\left(\widetilde{\boldsymbol{u}}_{n}, \boldsymbol{v}\right)-j\left(\widetilde{\boldsymbol{u}}_{n}, \boldsymbol{u}_{n}\right) \\
\geq \ell\left(\boldsymbol{v}-\boldsymbol{u}_{n}\right)-d\left(\widetilde{\boldsymbol{u}}_{n}, \widetilde{\boldsymbol{u}}_{n}, \boldsymbol{v}-\boldsymbol{u}_{n}\right)
\end{array}\right.
$$

The variational problem (2.22) has a unique solution. Next, we define the mapping

$$
\begin{aligned}
& \Psi: \boldsymbol{W}_{n} \longrightarrow \boldsymbol{W}_{n} \\
& \widetilde{\boldsymbol{u}}_{n} \longrightarrow \boldsymbol{u}_{n}, \text { solution of (2.22). }
\end{aligned}
$$

It is apparent that $\boldsymbol{u}_{n}$ is the solution of (2.21) if and only if $\boldsymbol{u}_{n}$ is a fixed point of $\Psi$.

Step2. We take $\boldsymbol{v}=\mathbf{0}$ and $2 \boldsymbol{u}_{n}$ in (2.22) respectively. Adding the resulting inequalities, we find

$$
a\left(\boldsymbol{u}_{n}, \boldsymbol{u}_{n}\right)+j\left(\widetilde{\boldsymbol{u}}_{n}, \boldsymbol{u}_{n}\right)=\ell\left(\boldsymbol{u}_{n}\right)-d\left(\widetilde{\boldsymbol{u}}_{n}, \widetilde{\boldsymbol{u}}_{n}, \boldsymbol{u}_{n}\right),
$$

from which we deduce that $(j(\cdot, \cdot)$ being non-negative)

$$
\left\|\boldsymbol{u}_{n}\right\|_{1} \leq C\left(\|\boldsymbol{f}\|+\left\|\widetilde{\boldsymbol{u}}_{n}\right\|_{1}^{2}\right)=E,
$$

with $C$ a positive constant depending on $\nu$ and $\Omega$. Hence, $\Psi$ maps $\boldsymbol{W}_{n}$ into $\bar{B}\left(\boldsymbol{u}_{n}, E\right)$, a closed ball of centre $\boldsymbol{u}_{n}$ and radius $E$. Next, for the continuity of $\Psi$, we take $\widetilde{\boldsymbol{u}}_{n}, \widetilde{\boldsymbol{w}}_{n}$ in $\boldsymbol{W}_{n}$ such that $\Psi\left(\widetilde{\boldsymbol{u}}_{n}\right)=\boldsymbol{u}_{n}$ and $\Psi\left(\widetilde{\boldsymbol{w}}_{n}\right)=\boldsymbol{w}_{n}$. From the coercivity of $a(\cdot, \cdot)$ and $(2.22)$, one obtains

$$
\begin{aligned}
& 2 \nu C\left\|\boldsymbol{u}_{n}-\boldsymbol{w}_{n}\right\|_{1}^{2} \\
\leq & a\left(\boldsymbol{u}_{n}-\boldsymbol{w}_{n}, \boldsymbol{u}_{n}-\boldsymbol{w}_{n}\right) \\
= & a\left(\boldsymbol{u}_{n}, \boldsymbol{u}_{n}-\boldsymbol{w}_{n}\right)-a\left(\boldsymbol{w}_{n}, \boldsymbol{u}_{n}-\boldsymbol{w}_{n}\right) \\
\leq & j\left(\widetilde{\boldsymbol{u}}_{n}, \boldsymbol{w}_{n}\right)-j\left(\widetilde{\boldsymbol{u}}_{n}, \boldsymbol{u}_{n}\right)+j\left(\widetilde{\boldsymbol{w}}_{n}, \boldsymbol{u}_{n}\right)-j\left(\widetilde{\boldsymbol{w}}_{n}, \boldsymbol{w}_{n}\right) \\
& +d\left(\widetilde{\boldsymbol{u}}_{n}, \widetilde{\boldsymbol{u}}_{n}, \boldsymbol{w}_{n}-\boldsymbol{u}_{n}\right)-d\left(\widetilde{\boldsymbol{w}}_{n}, \widetilde{\boldsymbol{w}}_{n}, \boldsymbol{w}_{n}-\boldsymbol{u}_{n}\right) \\
= & j\left(\widetilde{\boldsymbol{u}}_{n}, \boldsymbol{w}_{n}\right)-j\left(\widetilde{\boldsymbol{u}}_{n}, \boldsymbol{u}_{n}\right)+j\left(\widetilde{\boldsymbol{w}}_{n}, \boldsymbol{u}_{n}\right)-j\left(\widetilde{\boldsymbol{w}}_{n}, \boldsymbol{w}_{n}\right) \\
& +d\left(\widetilde{\boldsymbol{u}}_{n}-\widetilde{\boldsymbol{w}}_{n}, \widetilde{\boldsymbol{u}}_{n}, \boldsymbol{w}_{n}-\boldsymbol{u}_{n}\right)-d\left(\widetilde{\boldsymbol{w}}_{n}, \widetilde{\boldsymbol{w}}_{n}-\widetilde{\boldsymbol{u}}_{n}, \boldsymbol{w}_{n}-\boldsymbol{u}_{n}\right) \\
\leq & \kappa C_{0}\left\|\widetilde{\boldsymbol{u}}_{n}-\widetilde{\boldsymbol{u}}_{n}\right\|_{1}\left\|\boldsymbol{w}-\boldsymbol{u}_{n}\right\|_{1}+C\left(\left\|\widetilde{\boldsymbol{u}}_{n}\right\|_{1}+\left\|\widetilde{\boldsymbol{w}}_{n}\right\|_{1}\right)\left\|\boldsymbol{w}_{n}-\boldsymbol{u}_{n}\right\|_{1}\left\|\widetilde{\boldsymbol{w}}_{n}-\widetilde{\boldsymbol{u}}_{n}\right\|_{1} .
\end{aligned}
$$

Hence $\Psi$ is continuous. The Brouwer's fixed point theorem (see [19], Chap. IV, corollary 1.1 for instance) yields that, for each $n$, there exists a $\boldsymbol{u}_{n}$ satisfying (2.21), and

$$
\left\|\boldsymbol{u}_{n}\right\|_{1} \leq C\|\boldsymbol{f}\|
$$


Step 3. From (2.25) and owing to the compactness of the imbedding of $H^{1}(\Omega)$ into $L^{4}(\Omega)$, there exists a subsequence, still denoted by $\boldsymbol{u}_{n}$, which converges to $\boldsymbol{u}$ weakly in $\boldsymbol{H}^{1}(\Omega)$ and strongly in $\boldsymbol{L}^{4}(\Omega)$. Next, for $m \leq n$, it is noted that $\boldsymbol{u}_{n}$ satisfy also $(2.21)$.

Passing to the limit on $n$ is direct for the linear term and follows from the strong convergence in $\boldsymbol{L}^{4}(\Omega)$ for the terms $\left(\boldsymbol{u}_{n} \cdot \nabla\right) \boldsymbol{u}_{n}$. Next, one sees that the mapping $\boldsymbol{v} \rightarrow j(\boldsymbol{v}, \boldsymbol{v})$ is convex and lower semi-continuous. Hence

$$
j(\boldsymbol{u}, \boldsymbol{u}) \leq \liminf j\left(\boldsymbol{u}_{n}, \boldsymbol{u}_{n}\right) .
$$

Finally following G. Duvaut and J.L. Lions [8], we derive that $\boldsymbol{u}$ satisfies the limiting equation

$$
\left\{\begin{array}{l}
\text { for all } \boldsymbol{v} \in \boldsymbol{V}_{\mathrm{div}}, \\
a(\boldsymbol{u}, \boldsymbol{v}-\boldsymbol{u})+d(\boldsymbol{u}, \boldsymbol{u}, \boldsymbol{v}-\boldsymbol{u})+j(\boldsymbol{u}, \boldsymbol{v})-j(\boldsymbol{u}, \boldsymbol{u}) \geq \ell(\boldsymbol{v}-\boldsymbol{u}) .
\end{array}\right.
$$

The fact that $\boldsymbol{u}$ is in $\boldsymbol{V}_{\text {div }}$ comes from the observation that $\boldsymbol{V}_{\text {div }}$ is closed and $\boldsymbol{u}_{n} \in \boldsymbol{V}_{\mathrm{div}}$.

The uniqueness of solutions is achieved by assuming first the existence of two such solutions and then use standard properties on $a(\cdot, \cdot), d(\cdot, \cdot, \cdot)$ and $j(\cdot, \cdot)$.

The following result is a reformulation of (2.17) with a "Lagrange multiplier".

Lemma 2.2. Define the closed and convex set

$$
\mathcal{K}=\left\{\boldsymbol{\alpha} \in \boldsymbol{L}^{2}(S), \quad|\boldsymbol{\alpha}| \leq 1 \text { a.e. on } S\right\} .
$$

Let $(\boldsymbol{u}, p) \in \boldsymbol{V} \times M$. Then $(\boldsymbol{u}, p)$ is the solution of (2.17) if and only if there exists $\boldsymbol{\alpha} \in \mathcal{K}$ such that

$$
\left\{\begin{array}{l}
\forall(\boldsymbol{v}, q) \in \boldsymbol{V} \times M, \\
a(\boldsymbol{u}, \boldsymbol{v})+d(\boldsymbol{u}, \boldsymbol{u}, \boldsymbol{v})+b(\boldsymbol{v}, p)+\left(g+\kappa\left|\boldsymbol{u}_{\boldsymbol{\tau}}\right|, \boldsymbol{\alpha} \cdot \boldsymbol{v}_{\boldsymbol{\tau}}\right)_{S}=\ell(\boldsymbol{v}), \\
b(\boldsymbol{u}, q)=0 \\
\boldsymbol{\alpha} \cdot \boldsymbol{u}_{\boldsymbol{\tau}}=\left|\boldsymbol{u}_{\boldsymbol{\tau}}\right| \text { a.e. on } S .
\end{array}\right.
$$

Remark 2.1. Lemma 2.2 can be proved using either the Hahn-Banach Theorem [8], or one makes use of a more constructive approach based on regularization [20].

One may also observe that the fourth relation in (2.27) is equivalent to

$$
\boldsymbol{\alpha}=\mathcal{P}_{\mathcal{K}}\left[\boldsymbol{\alpha}+r\left(g+\kappa\left|\boldsymbol{u}_{\boldsymbol{\tau}}\right|\right) \boldsymbol{u}_{\boldsymbol{\tau}}\right], \quad \forall r>0,
$$


with $\mathcal{P}_{\mathcal{K}}: \boldsymbol{L}^{2}(S) \longrightarrow \mathcal{K}$ defined by

$$
\mathcal{P}_{\mathcal{K}}(\gamma)(x)=\frac{\gamma(x)}{\max (1,|\gamma(x)|)} \text {, a.e. on } S \text {, for all } \boldsymbol{\gamma} \in \boldsymbol{L}^{2}(S) \text {. }
$$

In [7], the authors have formulated the Uzawa's method based on (2.28) (see also [25, 20] where many more examples are presented based on this kind of characterization of "Lagrange multiplier"). One may argue that $\boldsymbol{\alpha}$ defined in Lemma 2.2 is not exactly a Lagrange nor Kuhn-Tucker multiplier, but obviously it has some common properties with such vectors. Hence it is called a multiplier in many publications.

\section{Alternating direction method of multipliers: Stokes System}

\subsection{Augmented Lagrangian formulation}

For $\boldsymbol{v}$ element of $\boldsymbol{V}$, and $\phi$ defined on $S$ such that $j(\phi, \phi)$ makes sense, we let

$$
\boldsymbol{F}(\boldsymbol{v}, \boldsymbol{\phi})=\frac{1}{2} a(\boldsymbol{v}, \boldsymbol{v})-\ell(\boldsymbol{v})+j(\boldsymbol{\phi}, \boldsymbol{\phi}) .
$$

It is clear that (2.13) is equivalent to the following constrained minimization problem

$$
\left\{\begin{array}{l}
\text { Find }(\boldsymbol{u}, \boldsymbol{\phi}) \in \boldsymbol{V}_{\mathrm{div}} \times \boldsymbol{L}^{2}(S) \text { such that } \\
\boldsymbol{F}(\boldsymbol{u}, \boldsymbol{\phi}) \leq \boldsymbol{F}(\boldsymbol{v}, \boldsymbol{\psi}) \quad \forall(\boldsymbol{v}, \boldsymbol{\psi}) \in \boldsymbol{V}_{\mathrm{div}} \times \boldsymbol{L}^{2}(S) \\
\boldsymbol{v}_{\boldsymbol{\tau}}-\boldsymbol{\phi}=0 \text { on } S
\end{array}\right.
$$

We can associate with (3.2) the augmented Lagrangian functional $\mathcal{L}_{r}: \boldsymbol{V}_{\text {div }} \times \boldsymbol{L}^{2}(S) \times \boldsymbol{L}^{2}(S) \longmapsto \mathbb{R}$ defined as follows

$$
\begin{aligned}
& \mathcal{L}_{r}(\boldsymbol{v}, \boldsymbol{\psi} ; \boldsymbol{\mu})=J_{0}(\boldsymbol{v})+j(\boldsymbol{\psi}, \boldsymbol{\psi})+\left(\boldsymbol{\mu}, \boldsymbol{v}_{\boldsymbol{\tau}}-\boldsymbol{\psi}\right)_{S}+\frac{r}{2}\left\|\boldsymbol{v}_{\boldsymbol{\tau}}-\boldsymbol{\psi}\right\|_{L^{2}(S)}^{2}, \\
& J_{0}(\boldsymbol{v})=\frac{1}{2} a(\boldsymbol{v}, \boldsymbol{v})-\ell(\boldsymbol{v})
\end{aligned}
$$

where $\boldsymbol{\mu}$ is a Lagrange multiplier associated with the second equation in (3.2) and $r>0$ is the penalty parameter. The corresponding saddle-point problem is

$$
\left\{\begin{array}{l}
\text { Find }(\boldsymbol{u}, \boldsymbol{\phi} ; \boldsymbol{\lambda}) \in \boldsymbol{V}_{\mathrm{div}} \times \boldsymbol{L}^{2}(S)^{2} \text { such that } \\
\text { for all }(\boldsymbol{v}, \boldsymbol{\psi} ; \boldsymbol{\mu}) \in \boldsymbol{V}_{\mathrm{div}} \times \boldsymbol{L}^{2}(S)^{2} \\
\mathcal{L}_{r}(\boldsymbol{u}, \boldsymbol{\phi} ; \boldsymbol{\mu}) \leq \mathcal{L}_{r}(\boldsymbol{u}, \boldsymbol{\phi} ; \boldsymbol{\lambda}) \leq \mathcal{L}_{r}(\boldsymbol{v}, \boldsymbol{\psi} ; \boldsymbol{\lambda})
\end{array}\right.
$$

Following [20] (page 168, Theorem 2.1), one can claim that 
Lemma 3.1. Let $r>0$, then $\boldsymbol{u}$ is the minimizer of $J_{\boldsymbol{s}}$ if and only if $(\boldsymbol{u}, \boldsymbol{\phi}, \boldsymbol{\lambda})$ is the saddle point of $\mathcal{L}_{r}$ with $\boldsymbol{\phi}=\boldsymbol{u}_{\boldsymbol{\tau}}$.

Remark 3.1. Since $(\boldsymbol{u}, \boldsymbol{\phi}, \boldsymbol{\lambda}) \in \boldsymbol{V}_{\mathrm{div}} \times \boldsymbol{L}^{2}(S) \times \boldsymbol{L}^{2}(S)$ is the solution of

$$
\mathcal{L}_{r}(\boldsymbol{u}, \boldsymbol{\phi} ; \boldsymbol{\lambda}) \leq \mathcal{L}_{r}(\boldsymbol{v}, \boldsymbol{\psi} ; \boldsymbol{\lambda}) \text { for all }(\boldsymbol{v}, \boldsymbol{\psi}) \in \boldsymbol{V}_{\mathrm{div}} \times \boldsymbol{L}^{2}(S),
$$

we then deduce that $(\boldsymbol{u}, \boldsymbol{\phi})$ is characterized by the following relations (see Lemma 2.1)

$$
\left\{\begin{array}{l}
\text { for all }(\boldsymbol{v}, \boldsymbol{\psi}) \in \boldsymbol{V}_{\mathrm{div}} \times \boldsymbol{L}^{2}(S), \\
a(\boldsymbol{u}, \boldsymbol{v})+r\left(\boldsymbol{u}_{\boldsymbol{\tau}}, \boldsymbol{v}_{\boldsymbol{\tau}}\right)_{S}=\ell(\boldsymbol{v})-\left(\boldsymbol{\lambda}, \boldsymbol{v}_{\boldsymbol{\tau}}\right)_{S}+r\left(\boldsymbol{\phi}, \boldsymbol{v}_{\boldsymbol{\tau}}\right)_{S}, \\
(r+2 \kappa)(\boldsymbol{\phi}, \boldsymbol{\psi}-\boldsymbol{\phi})_{S}+(g,|\boldsymbol{\psi}|-|\boldsymbol{\phi}|)_{S} \geq\left(\lambda+r \boldsymbol{u}_{\boldsymbol{\tau}}, \boldsymbol{\psi}-\boldsymbol{\phi}\right)_{S} .
\end{array}\right.
$$

\subsection{Alternating direction method of multipliers}

The alternating direction method of multipliers (ADMM) has been used in nonlinear mechanics (sometimes under the name of ALG2) to implement operator-splitting and domain decomposition methods [10, 23, 25, 32, 34, 33]. Applying ADMM to the saddle-point problem (3.4), leads to the following iterative process (starting with $\phi^{0}$ and $\lambda^{0}$ )

$$
\begin{aligned}
\boldsymbol{u}^{n+1} & =\arg \min _{\boldsymbol{u} \in \boldsymbol{V}_{\mathrm{div}}} \mathcal{L}_{r}\left(\boldsymbol{u}, \boldsymbol{\phi}^{n}, \boldsymbol{\lambda}^{n}\right), \\
\boldsymbol{\phi}^{n+1} & =\arg \min _{\boldsymbol{\phi} \in \boldsymbol{L}^{2}(S)} \mathcal{L}_{r}\left(\boldsymbol{u}^{n+1}, \boldsymbol{\phi}, \boldsymbol{\lambda}^{n}\right), \\
\boldsymbol{\lambda}^{n+1} & =\boldsymbol{\lambda}^{n}+r\left(\boldsymbol{u}_{\boldsymbol{\tau}}^{n+1}-\boldsymbol{\phi}^{n+1}\right) .
\end{aligned}
$$

Now, re-interpreting (3.6) in view of the first equation in (3.5), one obtains

$$
a\left(\boldsymbol{u}^{n+1}, \boldsymbol{v}\right)+r\left(\boldsymbol{u}_{\boldsymbol{\tau}}^{n+1}, \boldsymbol{v}_{\boldsymbol{\tau}}\right)_{S}=\ell(\boldsymbol{v})+\left(r \boldsymbol{\phi}^{n}-\boldsymbol{\lambda}^{n}, \boldsymbol{v}_{\boldsymbol{\tau}}\right)_{S}, \quad \forall \boldsymbol{v} \in \boldsymbol{V}_{\mathrm{div}} .
$$

We let

$$
\ell_{1}(\boldsymbol{v})=\ell(\boldsymbol{v})+\left(r \boldsymbol{\phi}^{n}-\boldsymbol{\lambda}^{n}, \boldsymbol{v}_{\boldsymbol{\tau}}\right)_{S}
$$

Using the divergence constraint in $\boldsymbol{V}_{\text {div }}$ explicitly, we get the Stokes-like problem

$$
\left\{\begin{array}{l}
\text { Find }\left(\boldsymbol{u}^{n+1}, p^{n+1}\right) \in \boldsymbol{V} \times M \text { such that, } \\
a\left(\boldsymbol{u}^{n+1}, \boldsymbol{v}\right)+r\left(\boldsymbol{u}_{\boldsymbol{\tau}}^{n+1}, \boldsymbol{v}_{\boldsymbol{\tau}}\right)_{S}+b\left(\boldsymbol{v}, p^{n+1}\right)=\ell_{1}(\boldsymbol{v}), \\
b\left(\boldsymbol{u}^{n+1}, q\right)=0 \\
\text { for all }(\boldsymbol{v}, q) \in \boldsymbol{V} \times M
\end{array}\right.
$$

Next, from (3.7) and the second equation of (3.5), one has $(r+2 \kappa)\left(\boldsymbol{\phi}^{n+1}, \boldsymbol{\psi}-\boldsymbol{\phi}^{n+1}\right)_{S}+\left(g,|\boldsymbol{\psi}|-\left|\boldsymbol{\phi}^{n+1}\right|\right)_{S} \geq\left(\lambda^{n}+r \boldsymbol{u}_{\boldsymbol{\tau}}^{n+1}, \boldsymbol{\psi}-\boldsymbol{\phi}^{n+1}\right)_{S}$, 
which is equivalent to the minimization problem

$$
\left\{\begin{array}{l}
\text { Find } \boldsymbol{\phi}^{n+1} \in L^{2}(S) \text { such that } \\
G\left(\boldsymbol{\phi}^{n+1}\right) \leq G(\boldsymbol{\psi}) \text { for all } \boldsymbol{\psi} \in L^{2}(S), \\
G(\boldsymbol{\psi})=\frac{r+2 k}{2}\|\boldsymbol{\psi}\|_{L^{2}(S)}^{2}+(g,|\boldsymbol{\psi}|)_{S}-\left(\boldsymbol{\lambda}^{n}+r \boldsymbol{u}_{\boldsymbol{\tau}}^{n+1}, \boldsymbol{\psi}\right)_{S}
\end{array}\right.
$$

We next want to compute the unknown $\phi^{n+1}$ realizing (3.11). For that purpose, we need some elements of the duality theory due to Fenchel (the interested reader can also consult [9]). So decompose the functional $G(\cdot)$ by introducing the functionals $\mathcal{F}$ and $\mathcal{G}$ defined on $\boldsymbol{X}=\boldsymbol{L}^{2}(S)$ as follows

$$
\begin{aligned}
\mathcal{F}(\phi) & =\frac{r+2 \kappa}{2}\|\phi\|_{S}^{2}-\left(\boldsymbol{\lambda}^{n}+r \boldsymbol{u}_{\boldsymbol{\tau}}^{n+1}, \phi\right)_{S}, \\
\mathcal{G}(\boldsymbol{\psi}) & =\int_{S} g|\boldsymbol{\psi}| d \Gamma .
\end{aligned}
$$

Using $\Lambda=$ Id (the identity operator), the minimization problem (3.11) can be rewritten as

$$
\inf _{\phi \in \boldsymbol{X}}\{\mathcal{F}(\phi)+\mathcal{G}(\Lambda \psi)\}
$$

for which the Fenchel dual problem is

$$
\sup _{\boldsymbol{y}^{*} \in \boldsymbol{X}^{*}}-\mathcal{F}^{*}\left(-\boldsymbol{y}^{*}\right)-\mathcal{G}\left(\boldsymbol{y}^{*}\right),
$$

where $\mathcal{F}^{*}: \boldsymbol{X}^{*}=\boldsymbol{X} \rightarrow \mathbb{R} \cup\{+\infty\}, \mathcal{G}^{*}: \boldsymbol{X}^{*}=\boldsymbol{X} \rightarrow \mathbb{R} \cup\{+\infty\}$ denote the convex conjugate of $\mathcal{F}$ and $\mathcal{G}$, respectively. In (3.15), $\boldsymbol{y}^{*}$ is called the dual variable. The convex conjugate functionals are given by

$$
\begin{aligned}
\mathcal{F}^{*}\left(\phi^{*}\right) & =\sup _{\boldsymbol{\phi}}\left\{\left(\phi^{*}, \phi\right)-\mathcal{F}(\phi)\right\} \\
& =\frac{1}{2(r+2 k)}\left\|\boldsymbol{\phi}^{*}+\boldsymbol{\lambda}^{n}+r \boldsymbol{u}_{\boldsymbol{\tau}}^{n+1}\right\|_{S}, \\
\mathcal{G}^{*}\left(\boldsymbol{\psi}^{*}\right) & =\sup _{\boldsymbol{\psi}}\left(\boldsymbol{\psi}^{*}, \boldsymbol{\psi}\right)-\mathcal{F}(\boldsymbol{\psi}) \\
& =\left\{\begin{array}{ll}
0 & \text { if }\left|\boldsymbol{\psi}^{*}\right| \leq g \\
+\infty & \text { if }\left|\boldsymbol{\psi}^{*}\right|>g
\end{array} \quad \forall \boldsymbol{\psi}^{*} \in \boldsymbol{X} .\right.
\end{aligned}
$$

Then the corresponding Fenchel dual problem is

$$
\sup _{\left|\boldsymbol{y}^{*}\right| \leq g}-\frac{1}{2(r+2 k)}\left\|-\boldsymbol{y}^{*}+\boldsymbol{\lambda}^{n}+r \boldsymbol{u}_{\boldsymbol{\tau}}^{n+1}\right\|_{S}^{2}
$$

Since $\mathcal{F}$ is differentiable, the extremality condition between the primal solution $\phi^{n+1}$ and the dual solution $\overline{\boldsymbol{y}}^{*}$ is

$$
-\overline{\boldsymbol{y}}^{*}=\nabla \mathcal{F}\left(\phi^{n+1}\right)=(r+2 k) \boldsymbol{\phi}^{n+1}-\boldsymbol{\lambda}^{n}-r \boldsymbol{u}_{\boldsymbol{\tau}}^{n+1} .
$$


It follows that the primal solution is ( see e.g. [9] Chap 4.3)

$$
\begin{aligned}
\gamma^{n} & =\left|\boldsymbol{\lambda}^{n}+r \boldsymbol{u}_{\boldsymbol{\tau}}^{n+1}\right| \\
\boldsymbol{\phi}^{n+1} & = \begin{cases}\frac{\gamma^{n}-g}{(r+2 k) \gamma^{n}}\left(\boldsymbol{\lambda}^{n}+r \boldsymbol{u}_{\boldsymbol{\tau}}^{n+1}\right) & \text { if } \gamma^{n} \geq g, \\
0 & \text { if } \gamma^{n} \leq g .\end{cases}
\end{aligned}
$$

Gathering the results above, we obtain Algorithm 1.

Algorithm 1 Alternating direction method of multiplier (ADMM) for the Stokes problem with friction

Initialization $n=0 . r>0, \phi^{0}$ and $\boldsymbol{\lambda}^{0}$ are given.

Iteration $n \geq 0$. Compute successively $u^{n+1}, \phi^{n+1}$ and $\lambda^{n+1}$ as follows.

1. Find $\left(\boldsymbol{u}^{n+1}, p^{n+1}\right) \in \boldsymbol{V} \times L_{0}^{2}(\Omega)$ such that for all $(\boldsymbol{v}, q) \in \boldsymbol{V} \times$ $L^{2}(\Omega)$

$$
\begin{aligned}
a\left(\boldsymbol{u}^{n+1}, \boldsymbol{v}\right)+r\left(\boldsymbol{u}_{\boldsymbol{\tau}}^{n+1}, \boldsymbol{v}_{\boldsymbol{\tau}}\right)_{S}+b\left(\boldsymbol{v}, p^{n+1}\right) & =\ell_{1}(\boldsymbol{v}) \\
b\left(\boldsymbol{u}^{n+1}, q\right) & =0 .
\end{aligned}
$$

2. Setting $\gamma^{n}=\left|\lambda^{n}+r \boldsymbol{u}_{\tau}^{n+1}\right|$

$$
\boldsymbol{\phi}^{n+1}= \begin{cases}\frac{\gamma^{n}-g}{(r+2 k) \gamma^{n}}\left(\lambda^{n}+r \boldsymbol{u}_{\boldsymbol{\tau}}^{n+1}\right) & \text { if } \gamma^{n} \geq g, \\ 0 & \text { if } \gamma^{n} \leq g .\end{cases}
$$

3. Lagrange multiplier:

$$
\boldsymbol{\lambda}^{n+1}=\boldsymbol{\lambda}^{n}+r\left(\boldsymbol{u}_{\boldsymbol{\tau}}^{n+1}-\boldsymbol{\phi}^{n+1}\right)
$$

We iterate until the relative error on $\boldsymbol{u}^{n}$ and $\phi^{n}$ becomes sufficiently "small", i.e.

$$
\frac{\left\|\boldsymbol{u}^{n}-\boldsymbol{u}^{n-1}\right\|^{2}+\left\|\phi^{n}-\phi^{n-1}\right\|_{S}^{2}}{\left\|\boldsymbol{u}^{n}\right\|^{2}+\left\|\phi^{n}\right\|_{S}^{2}}<\epsilon^{2} .
$$

where $\epsilon$ is the tolerance. Algorithm 1 summarizes the different steps needed to compute the solution of the saddle point problem (3.4). Note that the linear system solved each iteration has a constant matrix. Then a complete or incomplete factorization can be performed once and for all at the initialization step.

Remark 3.2. It is noted that by [20, Theorem 5.1, Chap. 6], one can prove the convergence of Algorithm 1 provided that $\nu$ and $\kappa$ are chosen adequately. Algorithm 1 is equivalent to the standard operator-splitting algorithm ALG2 described, e.g., in [10] (Chap 3) or [23] (Chap 3). 


\section{Operator splitting for the solution of problem (NS)}

It should be noted that there are several challenges when one wants to solve numerically (2.17), namely;

(a) the nonlinear convection term $(\boldsymbol{u} \cdot \nabla) \boldsymbol{u}$,

(b) the frictional functional $j(\boldsymbol{u}, \boldsymbol{u})$ that brings inequality,

(c) the divergence constraint and related pressure.

In order to solve nonlinear elliptic variational inequality problems, several methods have been developed in the literature (see among them $[22,28,27]$ ). There are basically two approaches to overcome the difficulty mentioned in (b), namely the regularization and multiplier techniques. The regularization method consisting to "replace" the real formulation by a regularized one where the non-differentiable term (here $j$ ) is replaced by a smooth functional $j_{\epsilon}$, and in the process the inequality appearing in the real problem is transformed into an equality. In the case of "multiplier" method, a result such as Lemma 2.2 is used to introduce a new unknown at the expense of removing the inequality. In this work we will present an algorithm based in two step namely $[5,6]$ :

Step 1. Associate to (2.17) an initial value problem in $\boldsymbol{V} \times M$.

Step 2. Use operator-splitting to time discretize the above evolution problem.

\subsection{Evolution problem}

We obtain Step 1 by associating to (2.17) the following evolution problem

$$
\left\{\begin{array}{l}
\operatorname{problem}(\text { ENS }): \\
\text { Find }(\boldsymbol{u}(t), p(t)) \in \boldsymbol{V} \times M \text { such that for all }(\boldsymbol{v}, q) \in \boldsymbol{V} \times M, \\
\left\langle\partial_{t} \boldsymbol{u}(t), \boldsymbol{v}-\boldsymbol{u}(t)\right\rangle+a(\boldsymbol{u}(t), \boldsymbol{v}-\boldsymbol{u}(t))+d(\boldsymbol{u}(t), \boldsymbol{u}(t), \boldsymbol{v}-\boldsymbol{u}(t)) \\
+b(\boldsymbol{v}-\boldsymbol{u}(t), p(t))+j(\boldsymbol{u}(t), \boldsymbol{v})-j(\boldsymbol{u}(t), \boldsymbol{u}(t)) \geq \ell(\boldsymbol{v}-\boldsymbol{u}(t)), \\
b(\boldsymbol{u}(t), q)=0 \\
\boldsymbol{u}(0)=\boldsymbol{u}_{0}
\end{array}\right.
$$

The idea behind the consideration of (4.1) is to obtain the steady state of as $t \rightarrow \infty$. We then claim that

Theorem 4.1. Let $\widetilde{\boldsymbol{u}}$ be the solution of (2.17) and $\boldsymbol{u}$ the solution of (4.1). Then

$$
\|\boldsymbol{u}(t)-\widetilde{\boldsymbol{u}}\|^{2} \leq\left\|\boldsymbol{u}_{0}-\widetilde{\boldsymbol{u}}\right\|^{2} \exp \left[-C_{\Omega}\left(2 \nu-\frac{C_{\Omega}}{2 \nu}\|\boldsymbol{f}\|-C_{\Omega} \kappa\right) t\right] .
$$


If, moreover, $\nu$ and $\kappa$ are chosen in such a way that

$$
C_{\Omega}\left(\frac{\kappa}{\nu}+\frac{\|\boldsymbol{f}\|}{2 \nu^{2}}\right) \leq 2 .
$$

Then $\widetilde{\boldsymbol{u}}$ is the $L^{2}$-limit of $\boldsymbol{u}(t)$ as $t \rightarrow \infty$.

Proof. It suffices to observe that

$$
\begin{aligned}
& \frac{1}{2} \frac{d}{d t}\|\boldsymbol{u}-\widetilde{\boldsymbol{u}}\|^{2}+2 \nu\|\varepsilon(\widetilde{\boldsymbol{u}}-\boldsymbol{u})\|^{2} \\
\leq & d(\widetilde{\boldsymbol{u}}, \widetilde{\boldsymbol{u}}, \boldsymbol{u}-\widetilde{\boldsymbol{u}})+d(\boldsymbol{u}, \boldsymbol{u}, \widetilde{\boldsymbol{u}}-\boldsymbol{u})+j(\widetilde{\boldsymbol{u}}, \boldsymbol{u})-j(\widetilde{\boldsymbol{u}}, \widetilde{\boldsymbol{u}})+j(\boldsymbol{u}, \widetilde{\boldsymbol{u}})-j(\boldsymbol{u}, \boldsymbol{u}) \\
\leq & C_{\Omega}\|\varepsilon(\widetilde{\boldsymbol{u}})\|\|\varepsilon(\boldsymbol{u}-\widetilde{\boldsymbol{u}})\|^{2}+C_{\Omega} \kappa\|\varepsilon(\boldsymbol{u}-\widetilde{\boldsymbol{u}})\|^{2} .
\end{aligned}
$$

From Korn's inequality and integrating (4.3) with respect to time one gets the results announced.

Remark 4.1. (a) In order to solve (2.17), we solve (4.1) on the interval $(0, \infty)$ until one obtains a steady state. It should be noted that the $L^{2}$ convergence is exponential.

(b) The fact that the solution of (2.17) is unique implies that wherever we start the computation of solution of (4.1), as the time increases we will get to the steady state.

(c) It can be shown that the evolution problem (4.1) is well posed. (the interested reader may see [31]).

\subsection{An operator-splitting method: The Marchuk-Yanenko's scheme}

This subsection deals with the final step for computing the numerical solution of (2.17) via the fractional step or splitting up method. This paragraph borrow mainly from Chapter 6 of R. Glowinski [25], a treatise in computational science in which the Marchuk-Yanenko's algorithm is presented departing from the Lie's scheme. We recall that (4.1) can be written as the following initial value problem: Find $\phi$ such that

$$
\left\{\begin{array}{l}
\frac{d \phi}{d t}+\sum_{i=1}^{q} A_{i} \phi=0 \text { on }(0, T), \\
\phi(0)=\phi_{0} .
\end{array}\right.
$$

Let $k$ be the time discretization step, $t_{n}=n k$, and $\phi_{n} \approx \phi\left(t_{n}\right)$. Then Marchuk-Yanenko's method to solve (4.4) is as follows [25]:

$$
\phi^{0}=\phi_{0} ;
$$


then for $n \geq 1$, knowing $\phi_{n}$, and for $i=1,2,3, \cdots, q$ we compute $\phi^{n+1}$ via

$$
\frac{\phi^{n+i / q}-\phi^{n+(i-1) / q}}{k}+A_{i}\left(\phi^{n+i / q}, t^{n+1}\right)=0 .
$$

Hence adapting the algorithm (4.5) and (4.6), one has the following scheme: Starting with

$$
\boldsymbol{u}^{0}=\boldsymbol{u}_{0}
$$

for $n \geq 0$, with $t^{n+\alpha}=(n+\alpha) k$, and for non-negative $\gamma_{1}$ and $\gamma_{2}$, such that $\gamma_{1}+\gamma_{2}=1$, we compute $\boldsymbol{u}^{n+1 / 2}$ and then $\left(\boldsymbol{u}^{n+1}, p^{n+1}\right)$ as in Algorithm 2.

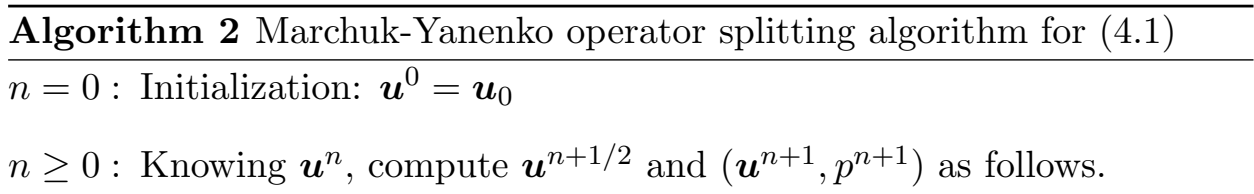

Step 1. Linear step without a constraint: compute $\boldsymbol{u}^{n+1 / 2}$ such that, for all $\boldsymbol{v} \in \boldsymbol{V}$

$$
\frac{1}{k}\left(\boldsymbol{u}^{n+1 / 2}-\boldsymbol{u}^{n}, \boldsymbol{v}\right)+\gamma_{1} a\left(\boldsymbol{u}^{n+1 / 2}, \boldsymbol{v}\right)+d\left(\boldsymbol{u}^{n}, \boldsymbol{u}^{n+1 / 2}, \boldsymbol{v}\right)=(\boldsymbol{f}, \boldsymbol{v}) .
$$

Step 2. Nonlinear elliptic variational inequality step with a constraint: compute $\left(\boldsymbol{u}^{n+1}, p^{n+1}\right)$ such that, for all $(\boldsymbol{v}, q) \in \boldsymbol{V} \times M$

$$
\begin{aligned}
\frac{1}{k}\left(\boldsymbol{u}^{n+1}-\boldsymbol{u}^{n+1 / 2}, \boldsymbol{v}-\boldsymbol{u}^{n+1}\right)+\gamma_{2} a\left(\boldsymbol{u}^{n+1}, \boldsymbol{v}\right. & \left.-\boldsymbol{u}^{n+1}\right) \\
+b\left(\boldsymbol{v}-\boldsymbol{u}^{n+1}, p^{n+1}\right)+j\left(\boldsymbol{u}^{n+1}, \boldsymbol{v}\right)-j\left(\boldsymbol{u}^{n+1}, \boldsymbol{u}^{n+1}\right) & \geq 0,(4.8) \\
b\left(\boldsymbol{u}^{n+1}, q\right) & =0 .(4.9)
\end{aligned}
$$

The Marchuk-Yanenko's splitting scheme allows us to decouple the following difficulties:

(1) The incompressibility condition and the related unknown pressure

(2) The advection terms

(3) The contact condition . 


\section{OPERATOR SPLITTING FOR THE SOLUTION OF PROBLEM (NS)17}

Remark 4.2. By definition, $b\left(\boldsymbol{u}^{n}, q\right)=0$ for all $n \geq 0$, while $b\left(\boldsymbol{u}^{n+1 / 2}, q\right)$ is not necessarily zero.

The sub-problem (4.7) is linear in $\boldsymbol{u}^{n+1 / 2}$. Hence its existence theory is a direct application of Lax-Milgram's Lemma. Indeed, the bilinear form

$$
(\boldsymbol{w}, \boldsymbol{v}) \longrightarrow \frac{1}{k}(\boldsymbol{w}, \boldsymbol{v})+\gamma_{1} a(\boldsymbol{w}, \boldsymbol{v})+d\left(\boldsymbol{u}^{n}, \boldsymbol{w}, \boldsymbol{v}\right)
$$

is continuous, and coercive on $\boldsymbol{V}$. The coercivity being obtained by the property of $d(\cdot, \cdot, \cdot)$. Next the functional

$$
\boldsymbol{v} \longrightarrow \frac{1}{k}\left(\boldsymbol{u}^{n}, \boldsymbol{v}\right)+(\boldsymbol{f}, \boldsymbol{v})
$$

is linear and continuous on $\boldsymbol{V}$.

The sub-problem (4.8)-(4.9) looks like problem (S) we dealt with in Section

2. Hence there is no need here to re-visit that sub-problem.

Concerning Algorithm 2, we have the following stability result

Theorem 4.2. Suppose that $\boldsymbol{u}_{0} \in \boldsymbol{L}^{2}(\Omega)$; then the scheme (4.7)-(4.9) is unconditionally stable in the following sense: for $n=0,1, \ldots, m$ there exists a positive constant $C_{\Omega}$ such that

$$
\begin{aligned}
& \left\|\boldsymbol{u}^{m}\right\|^{2} \leq\left\|\boldsymbol{u}_{0}\right\|^{2}+\frac{C_{\Omega} t_{m}}{\gamma_{1} \nu}\|\boldsymbol{f}\|^{2}, \\
& k \sum_{n=0}^{m-1}\left\|\varepsilon\left(\boldsymbol{u}^{n+1 / 2}\right)\right\|^{2} \leq \frac{1}{3 \nu \gamma_{1}}\left[\left\|\boldsymbol{u}_{0}\right\|^{2}+\frac{C_{\Omega} t_{m}}{\gamma_{1} \nu}\|\boldsymbol{f}\|^{2}\right] \\
& k \sum_{n=0}^{m-1}\left\|\varepsilon\left(\boldsymbol{u}^{n+1}\right)\right\|^{2} \leq \frac{1}{4 \nu \gamma_{2}}\left[\left\|\boldsymbol{u}_{0}\right\|^{2}+\frac{C_{\Omega} t_{m}}{\gamma_{1} \nu}\|\boldsymbol{f}\|^{2}\right] \\
& \sum_{n=0}^{m-1}\left\|\boldsymbol{u}^{n+1 / 2}-\boldsymbol{u}^{n}\right\|^{2} \leq\left\|\boldsymbol{u}_{0}\right\|^{2}+\frac{C_{\Omega} t_{m}}{\gamma_{1} \nu}\|\boldsymbol{f}\|^{2}, \\
& \sum_{n=0}^{m-1}\left\|\boldsymbol{u}^{n+1}-\boldsymbol{u}^{n+1 / 2}\right\|^{2} \leq\left\|\boldsymbol{u}_{0}\right\|^{2}+\frac{C_{\Omega} t_{m}}{\gamma_{1} \nu}\|\boldsymbol{f}\|^{2}, \\
& k \sum_{n=0}^{m-1} j\left(\boldsymbol{u}^{n+1}, \boldsymbol{u}^{n+1}\right) \leq \frac{1}{2}\left[\left\|\boldsymbol{u}_{0}\right\|^{2}+\frac{C_{\Omega} t_{m}}{\gamma_{1} \nu}\|\boldsymbol{f}\|^{2}\right] \\
& \left\|\boldsymbol{u}^{m+1 / 2}\right\|^{2} \leq\left\|\boldsymbol{u}_{0}\right\|^{2}+\frac{C_{\Omega} t_{m}}{\gamma_{1} \nu}\|\boldsymbol{f}\|^{2} .
\end{aligned}
$$

proof. Let $\boldsymbol{v}=\boldsymbol{u}^{n+1 / 2}$ in (4.7), one has

$$
2\left(\boldsymbol{u}^{n+1 / 2}-\boldsymbol{u}^{n}, \boldsymbol{u}^{n+1 / 2}\right)+2 k \gamma_{1} a\left(\boldsymbol{u}^{n+1 / 2}, \boldsymbol{u}^{n+1 / 2}\right)=2 k\left(\boldsymbol{f}, \boldsymbol{u}^{n+1 / 2}\right) .
$$




\section{OPERATOR SPLITTING FOR THE SOLUTION OF PROBLEM (NS)18}

The second term on the right hand side of (4.18) can be treated with CauchySchwarz, Korn and Young inequalities as follows

$$
\begin{aligned}
2 k\left(\boldsymbol{f}, \boldsymbol{u}^{n+1 / 2}\right) & \leq 2 C_{\Omega} k\|\boldsymbol{f}\|\left\|\boldsymbol{u}^{n+1 / 2}\right\| \\
& \leq 2 C_{\Omega} k\|\boldsymbol{f}\|\left\|\varepsilon\left(\boldsymbol{u}^{n+1 / 2}\right)\right\| \\
& \leq \frac{C_{\Omega} k}{\gamma_{1} \nu}\|\boldsymbol{f}\|^{2}+k \nu \gamma_{1}\left\|\varepsilon\left(\boldsymbol{u}^{n+1 / 2}\right)\right\|^{2} .
\end{aligned}
$$

Using (2.9) in (4.18) and having in mind (4.19), we find that

$$
\left\|\boldsymbol{u}^{n+1 / 2}\right\|^{2}-\left\|\boldsymbol{u}^{n}\right\|^{2}+\left\|\boldsymbol{u}^{n+1 / 2}-\boldsymbol{u}^{n}\right\|^{2}+3 \nu k \gamma_{1}\left\|\varepsilon\left(\boldsymbol{u}^{n+1 / 2}\right)\right\|^{2} \leq \frac{C_{\Omega} k}{\gamma_{1} \nu}\|\boldsymbol{f}\|^{2} .
$$

Next, we take successively $\boldsymbol{v}=0$ and $\boldsymbol{v}=\boldsymbol{u}^{n+1}$ in (4.9), and after comparison of the resulting relations and using (2.9), we find

$\left\|\boldsymbol{u}^{n+1}\right\|^{2}-\left\|\boldsymbol{u}^{n+1 / 2}\right\|^{2}+\left\|\boldsymbol{u}^{n+1}-\boldsymbol{u}^{n+1 / 2}\right\|^{2}+4 k \nu \gamma_{2}\left\|\varepsilon\left(\boldsymbol{u}^{n+1}\right)\right\|^{2}+2 k j\left(\boldsymbol{u}^{n+1}, \boldsymbol{u}^{n+1}\right)=0$.

We do $(4.20)+(4.21)$ for $n=0,1,2, \ldots, m-1$, we obtain

$$
\begin{aligned}
& \left\|\boldsymbol{u}^{m}\right\|^{2}+3 \nu k \gamma_{1} \sum_{n=0}^{m-1}\left\|\varepsilon\left(\boldsymbol{u}^{n+1 / 2}\right)\right\|^{2}+4 k \nu \gamma_{2} \sum_{n=0}^{m-1}\left\|\varepsilon\left(\boldsymbol{u}^{n+1}\right)\right\|^{2} \\
& +\sum_{n=0}^{m-1}\left\|\boldsymbol{u}^{n+1 / 2}-\boldsymbol{u}^{n}\right\|^{2}+\sum_{n=0}^{m-1}\left\|\boldsymbol{u}^{n+1}-\boldsymbol{u}^{n+1 / 2}\right\|^{2}+2 k \sum_{n=0}^{m-1} j\left(\boldsymbol{u}^{n+1}, \boldsymbol{u}^{n+1}\right) \\
& \leq\left\|\boldsymbol{u}_{0}\right\|^{2}+\frac{C_{\Omega} t_{m}}{\gamma_{1} \nu}\|\boldsymbol{f}\|^{2}
\end{aligned}
$$

from which we obtain $(4.11), \ldots,(4.16)$.

Next we add the relations (4.20) for $n=0,1,2, \ldots, m$ and dropping some positive terms, we obtain

$$
\sum_{n=0}^{m}\left\|\boldsymbol{u}^{n+1 / 2}\right\|^{2}-\sum_{n=0}^{m}\left\|\boldsymbol{u}^{n}\right\|^{2} \leq \frac{C_{\Omega} t_{m}}{\gamma_{1} \nu}\|\boldsymbol{f}\|^{2} .
$$

We add the relations (4.21) for $n=0,1,2, \ldots, m-1$ and dropping some positive terms, we obtain

$$
\sum_{n=0}^{m-1}\left\|\boldsymbol{u}^{n+1}\right\|^{2}-\sum_{n=0}^{m-1}\left\|\boldsymbol{u}^{n+1 / 2}\right\|^{2} \leq 0 .
$$

Adding (4.22) and (4.23) gives (4.17), which completes the proof of the theorem.

Remark 4.3. As mentioned before the sub problems (4.8)-(4.9) are in a way a modification of problem (S), which is treated in Section 3. 


\section{$5 \quad$ Finite element Approximations}

We assume that $\Omega$ is a bounded polygonal domain of $\mathbb{R}^{d}$, with $d=2,3$, and denote by $\mathcal{T}_{h}$ a conforming finite element triangulation of $\Omega$ (like those discussed in e.g., $[4,19])$. $\mathcal{P}_{l}(K)$ stands for the space of polynomials of degree less or equal to $l$ on $K$ the element of $\mathcal{T}_{h}$.

For our numerical simulations, we use stabilized $P_{1}-P_{1}$ pair of finite element, that is

$$
\begin{aligned}
& \boldsymbol{V}_{h}=\left\{\boldsymbol{v}_{h} \in \boldsymbol{V} \cap \mathcal{C}(\Omega)^{d},\left.\boldsymbol{v}_{h}\right|_{K} \in \mathcal{P}_{1}(K), \forall K \in \mathcal{T}_{h}\right\}, \\
& M_{h}=\left\{q_{h} \in M \cap \mathcal{C}(\Omega),\left.q_{h}\right|_{K} \in \mathcal{P}_{1}(K), \forall K \in \mathcal{T}_{h}\right\} .
\end{aligned}
$$

Stabilized methods for spatial discretization of the Stokes problem are very popular in engineering practice since they allow for the use of equal-order interpolation (the same mesh for velocity and pressure). Equal-order interpolation is very useful in large-scale multi-physics codes. Indeed, a code dealing with several independent variables (e.g. chemical species, velocity components, etc) requires the transfer of information between its different components.

The (global) stabilization is obtained by regularizing the discrete formulation of the Stokes problem, i.e.

Find $\left(\boldsymbol{u}_{h}, p_{h}\right) \in \boldsymbol{V}_{h} \times M_{h}$ such that, for all $\left(\boldsymbol{v}_{h}, q_{h}\right) \in \boldsymbol{V}_{h} \times M_{h}$

$$
\begin{aligned}
a\left(\boldsymbol{u}_{h}, \boldsymbol{v}_{h}-\boldsymbol{u}_{h}\right)-b\left(\boldsymbol{v}_{h}-\boldsymbol{u}_{h}, p_{h}\right)+j\left(\boldsymbol{u}_{h}, \boldsymbol{v}_{h}\right)-j\left(\boldsymbol{u}_{h}, \boldsymbol{u}_{h}\right) & \geq \ell\left(\boldsymbol{v}_{h}-\boldsymbol{u}_{h}\right), \\
-b\left(\boldsymbol{u}_{h}, q_{h}\right)-\beta c_{h}\left(p_{h}, q_{h}\right) & =0,
\end{aligned}
$$

where $\beta>0$ and $c_{h}$ is the scaled discrete diffusion operator $([29,41])$

$$
c_{h}\left(p_{h}, q_{h}\right)=\sum_{K \in \mathcal{T}_{h}} h_{K}^{2} \int_{K} \nabla p_{h} \cdot \nabla q_{h} \mathrm{~d} K,
$$

with $h_{K}$ the size of an element $K \in \mathcal{T}_{h}$. Numerical experiments suggest that the "optimal" value for $\beta$ close to $1 / 100$.

The discrete form of Algorithm 1, and Algorithm 2 are readily obtained with the obvious difference that the later are formulated in the discrete spaces $V_{h}$ and $M_{h}$.

\section{$6 \quad$ Numerical Simulations}

The algorithms outlined in the previous sections were implemented in MATLAB on a Linux workstation with $3.00 \mathrm{GHz}$ clock frequency and 32 GB RAM.

In Algorithm 1 we stop the iterations if the relative error on $\boldsymbol{u}^{k}$ and $\phi^{k}$ becomes sufficiently "small", i.e.

$$
\frac{\left\|\boldsymbol{u}^{k}-\boldsymbol{u}^{k-1}\right\|_{L^{2}}^{2}+\left\|\phi^{k}-\phi^{k-1}\right\|_{L^{2}}^{2}}{\left\|\boldsymbol{u}^{k}\right\|_{L^{2}}^{2}+\left\|\phi^{k}\right\|_{L^{2}}^{2}}<10^{-10} .
$$


To make sure that Algorithm 1 converges, we also check if the residual of the constraint is small, i.e., $\left\|\boldsymbol{u}^{k}-\boldsymbol{\phi}^{k}\right\|<10^{-4}$.

For the numerical solution of linear systems, we use two strategies.

- For 2D problems, a symmetric indefinite factorization function (using MATLAB's function ldl) is performed once and for all in the initialization step. Then in the rest of the iterative process, the solution of the linear systems is reduced to backward/forward substitutions.

- For 3D problems, an incomplete LU factorization (with $10^{-6}$ as drop tolerance) is performed and the result is used as preconditioner in the GMRES iterative solver.

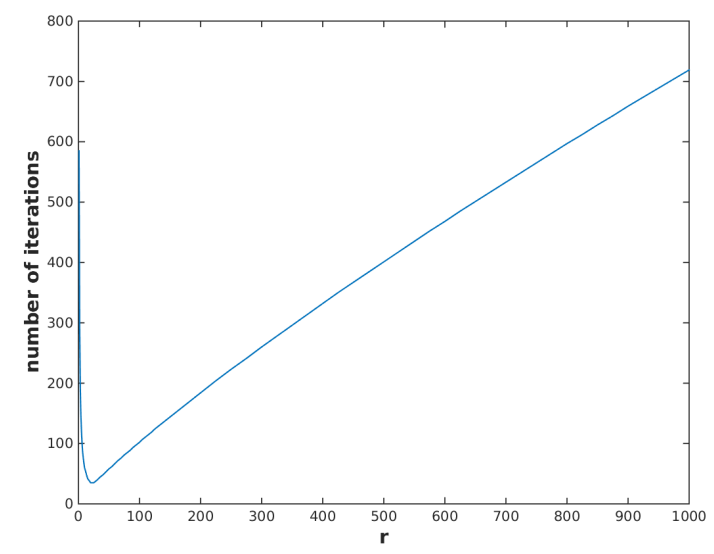

Figure 1: Number of iteration versus penalty parameter, $\kappa=0.1$ and $g=0.5$

\subsection{A two dimensional Stokes problem}

We consider the Stokes problem studied by J.K. Djoko and M. Mbehou in [7]. The flow domain is $\Omega=(0,1)^{2}, S=(0,1) \times\{1\}, \Gamma=\partial \Omega \backslash S, \alpha=0$, $\nu=1$ and the exact solution

$$
\begin{aligned}
u_{1}(x, y) & =20 x^{2}(1-x)^{2} y(1-2 y), \\
u_{2}(x, y) & =-20 x(1-x)(1-2 x)(1-y)^{2} y^{2}, \\
p(x, y) & =(2 x-1)(2 y-1) .
\end{aligned}
$$

The right-hand side in (2.1) is adjusted accordingly, i.e.

$$
\begin{aligned}
f_{1}(x, y)= & 80 x^{2}(1-x)^{2}-20\left(2+12 x^{2}-12 x\right) y(1-2 y)+2(2 y-1),(6.4) \\
f_{2}(x, y)= & 20(12 x-6) y^{2}(1-y)^{2}+20 x(1-2 x)(1-x)\left(2+12 y^{2}-12 y\right) \\
& +2(2 x-1) .
\end{aligned}
$$


Algorithm 1 is very sensitive to the choice of the penalty (or augmentation) parameter $r$. Figure 1 shows the number of iterations versus the penalty parameter for $h=1 / 16, \kappa=.1$ and $g=0.5$. The "optimal" penalty parameter is $r^{*} \approx 30$. Choosing larger values for $r$ increases the number of iterations without improving the final result. Figures 2-4 show velocity fields and streamlines for $g=0.5, g=1$ and $g=4$, respectively.
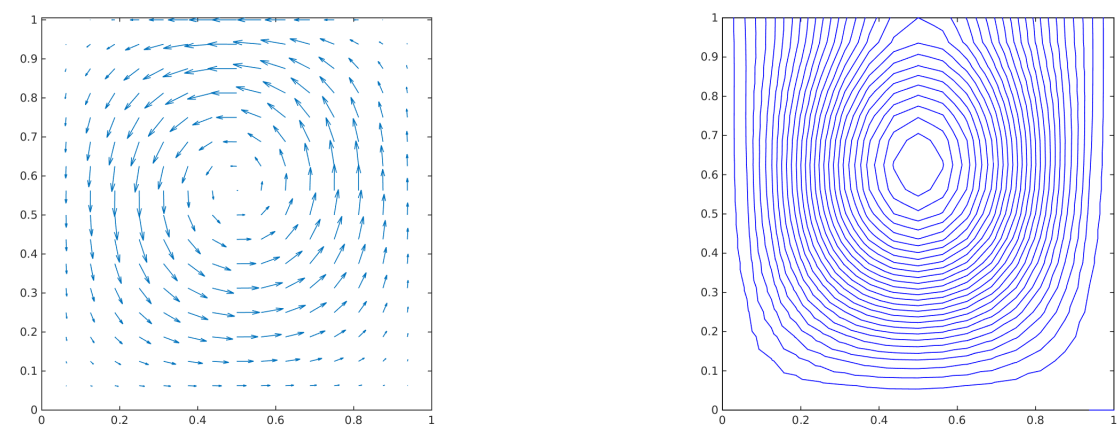

Figure 2: Velocity field and streamlines for $g=0.5$.
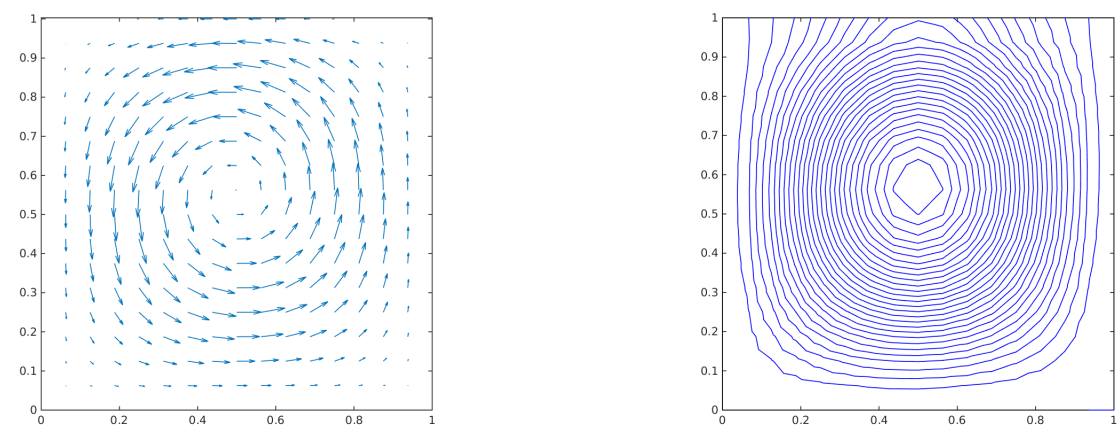

Figure 3: Velocity field and streamlines for $g=1$.

We now study the behavior of Algorithm 1. We report in Table 1 the number of iterations and CPU time of Algorithm 1 for different mesh sizes. We can notice that the number of iterations required for convergence is virtually independent of the mesh size. The convergence rates of the finite element approximation are evaluated in Table $2(g=0.5)$ and Table 3 $(g=1)$ as follows

$$
\begin{aligned}
e_{h}(\boldsymbol{u}) & =\left\|\boldsymbol{u}_{h}-\boldsymbol{u}_{*}\right\|_{L^{2}} \\
e_{h}(\boldsymbol{u}, p) & =\left\|\boldsymbol{u}_{h}-\boldsymbol{u}_{*}\right\|_{H^{1}}+\left\|p_{h}-p_{*}\right\|_{L^{2}}
\end{aligned}
$$

where $\left(\boldsymbol{u}_{*}, p_{*}\right)$ is the reference solution computed on a finer mesh with $h=$ $1 / 512$. 

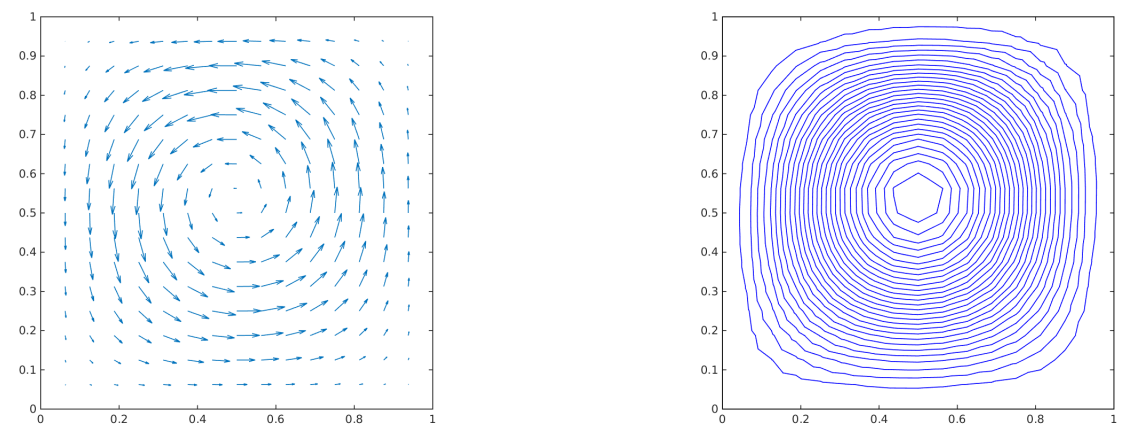

Figure 4: Velocity field and streamlines for $g=4$.

\begin{tabular}{|c|cc|cc|cc|}
\hline Mesh size $h$ & \multicolumn{2}{|c|}{$g=0.5$} & \multicolumn{2}{c|}{$g=1$} & \multicolumn{2}{c|}{$g=4$} \\
\hline & Iter & CPU (sec.) & Iter & CPU (sec.) & Iter & CPU (sec.) c \\
\hline $1 / 8$ & 36 & 0.05 & 32 & 0.066 & 22 & 0.047 \\
$1 / 16$ & 39 & 0.104 & 31 & 0.103 & 22 & 0.082 \\
$1 / 32$ & 38 & 0.260 & 32 & 0.280 & 21 & 0.203 \\
$1 / 64$ & 38 & 1.401 & 32 & 1.232 & 11 & 0.702 \\
$1 / 128$ & 39 & 10.063 & 33 & 9.041 & 10 & 4.574 \\
\hline
\end{tabular}

Table 1: Performances of Algorithm 1 with $r=r^{*}, \kappa=.1$.

\begin{tabular}{|ccccc|}
\hline$h$ & $e_{h}(\boldsymbol{u})$ & rate & $e_{h}(\boldsymbol{u}, p)$ & rate \\
\hline $1 / 8$ & $1.054 \times 10^{-2}$ & - & $2.649 \times 10^{-1}$ & - \\
$1 / 16$ & $2.838 \times 10^{-3}$ & 1.89 & $9.320 \times 10^{-2}$ & 1.50 \\
$1 / 32$ & $6.959 \times 10^{-4}$ & 2.02 & $3.319 \times 10^{-2}$ & 1.48 \\
$1 / 64$ & $1.706 \times 10^{-4}$ & 2.02 & $1.230 \times 10^{-2}$ & 1.43 \\
$1 / 128$ & $4.019 \times 10^{-5}$ & 2.08 & $4.706 \times 10^{-3}$ & 1.38 \\
\hline
\end{tabular}

Table 2: Convergence rates for Algorithm 1 with $r=r^{*}, \kappa=0.1$ and $g=0.5$.

\begin{tabular}{|c|cccc|}
\hline$h$ & $e_{h}(\boldsymbol{u})$ & rate & $e_{h}(\boldsymbol{u}, p)$ & rate \\
\hline $1 / 8$ & $1.018 \times 10^{-2}$ & - & $2.766 \times 10^{-1}$ & - \\
$1 / 16$ & $2.571 \times 10^{-3}$ & 1.98 & $9.415 \times 10^{-2}$ & 1.55 \\
$1 / 32$ & $6.724 \times 10^{-4}$ & 1.93 & $3.398 \times 10^{-2}$ & 1.47 \\
$1 / 64$ & $1.610 \times 10^{-4}$ & 2.06 & $1.270 \times 10^{-2}$ & 1.41 \\
$1 / 128$ & $3.821 \times 10^{-5}$ & 2.07 & $4.821 \times 10^{-3}$ & 1.39 \\
\hline
\end{tabular}

Table 3: Convergence rates for Algorithm 1 with $r=r^{*}, \kappa=0.1$ and $g=1$. 


\begin{tabular}{|c|cccc|}
\hline$h$ & $e_{h}(\boldsymbol{u})$ & rate & $e_{h}(\boldsymbol{u}, p)$ & rate \\
\hline $1 / 8$ & $8.975 \times 10^{-3}$ & - & $2.805 \times 10^{-1}$ & - \\
$1 / 16$ & $2.307 \times 10^{-3}$ & 1.95 & $9.812 \times 10^{-2}$ & 1.51 \\
$1 / 32$ & $5.696 \times 10^{-4}$ & 2.01 & $3.395 \times 10^{-2}$ & 1.53 \\
$1 / 64$ & $1.388 \times 10^{-4}$ & 2.03 & $1.230 \times 10^{-2}$ & 1.46 \\
$1 / 128$ & $3.270 \times 10^{-5}$ & 2.08 & $4.564 \times 10^{-3}$ & 1.43 \\
\hline
\end{tabular}

Table 4: Convergence rates for Algorithm 1 with $r=r^{*}, \kappa=0.1$ and $g=4$.

\subsection{A two dimensional Navier-Stokes problem}

For the Navier-Stokes problem we consider the exact solution (6.1)-(6.3) with the same values of $\nu, g, \kappa$ and we adjust the right-hand side accordingly, i.e.

$$
\boldsymbol{f}=\boldsymbol{f}_{\text {Stokes }}+(\boldsymbol{u} \cdot \nabla) \boldsymbol{u} .
$$

In the time stepping scheme, Algorithm 2, we set $\gamma_{1}=\gamma_{2}=1 / 2$, and $k=1 / 100$. We use Algorithm 1 as solver (4.8)-(4.9). Since we use the same values (for $\nu, \kappa$ and $g$ ) as in the Stokes case, we keep the "optimal" penalty parameter $r^{*} \approx 30$. At each time step, Algorithm 1 is initialized with the solution $\left(\boldsymbol{u}^{n}, \phi^{n}, \boldsymbol{\lambda}^{n}\right)$ obtained at the previous time step. With this strategy, Algorithm 1 converges in less than 10 iterations. We assume that a stationary solution is reached if the relative $L^{2}(\Omega)$-error for $\left(\boldsymbol{u}_{h}, p_{h}\right)$ is less than $10^{-6}$.

Figures (5)-(7) show the velocity fields and the streamlines in $\Omega$ for $g=.5, g=1$ and $g=4$, respectively. We observe similar pattern as in the Stokes case.
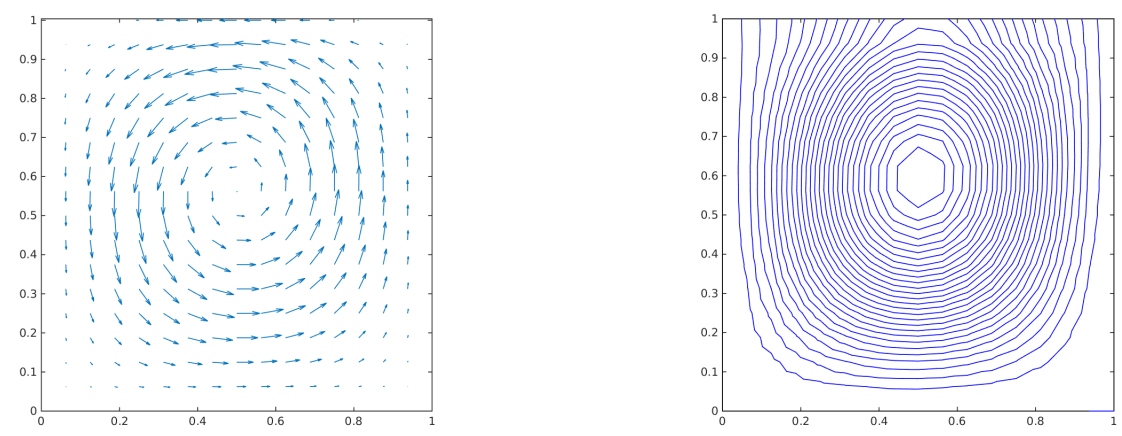

Figure 5: Velocity field and streamlines for $g=0.5$.

In Table 5 we report the behavior of the Marchuk-Yanenko's scheme in terms of number of iterations required to reach a stationary solution and the corresponding CPU time. As expected, the computational times are longer for the Navier-Stokes case. In Table 6-8, we report the convergence rates of the finite element approximation, computed using a reference solution as in the Stokes case (Section 6.1). 

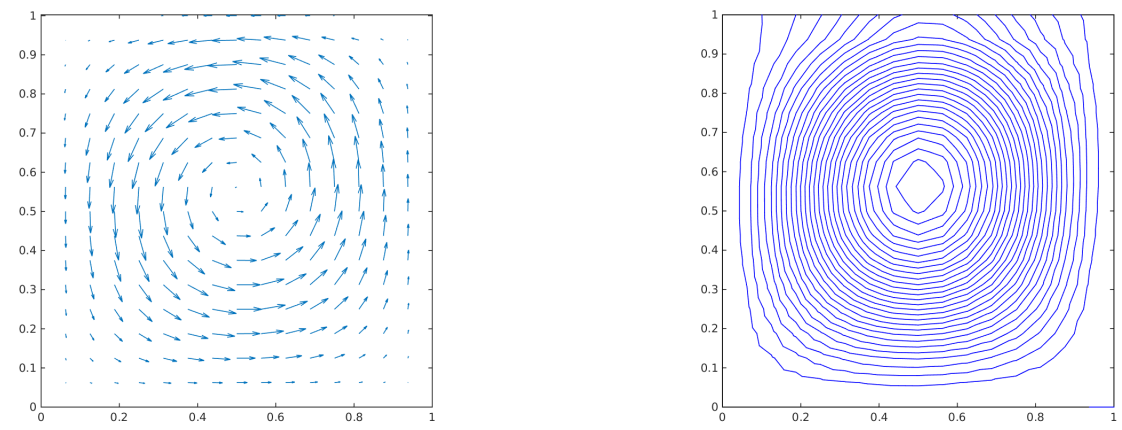

Figure 6: Velocity field and streamlines for $g=1$.
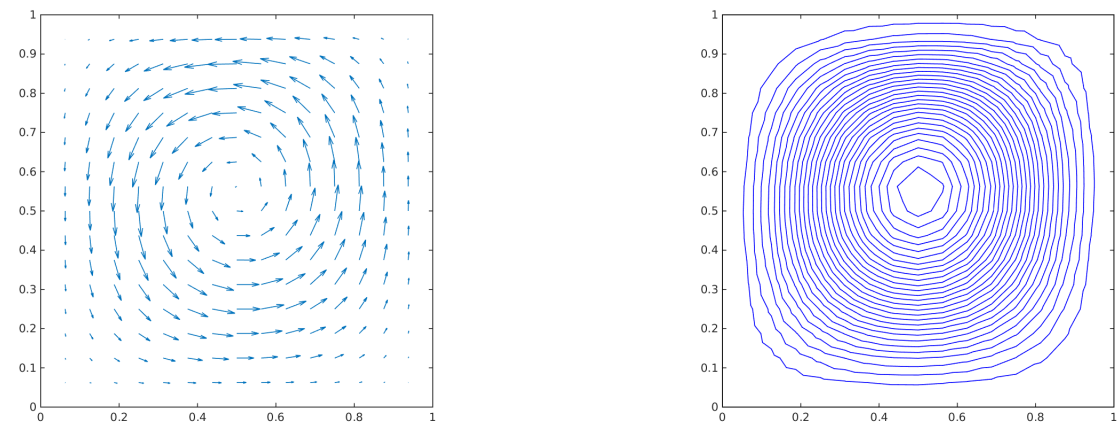

Figure 7: Velocity field and streamlines for $g=4$.

\begin{tabular}{|c|cc|cc|cc|}
\hline Mesh size $h$ & \multicolumn{2}{|c|}{$g=0.5$} & \multicolumn{2}{c|}{$g=1$} & \multicolumn{2}{c|}{$g=4$} \\
\hline & Iter & CPU (sec.) & Iter & CPU (sec.) & Iter & CPU (sec.) \\
\hline $1 / 8$ & 230 & 0.511 & 290 & 0.439 & 280 & 0.668 \\
$1 / 16$ & 330 & 1.345 & 300 & 0.712 & 290 & 0.582 \\
$1 / 32$ & 340 & 5.319 & 310 & 2.411 & 300 & 1.604 \\
$1 / 64$ & 340 & 29.731 & 310 & 14.560 & 300 & 7.837 \\
$1 / 128$ & 340 & 247.452 & 310 & 116.080 & 300 & 59.536 \\
\hline
\end{tabular}

Table 5: Number of iterations required for a stationary solution and CPU times (in seconds) for the Navier-Stokes problem with (6.6) .

\begin{tabular}{|ccccc|}
\hline$h$ & $e_{h}(\boldsymbol{u})$ & rate & $e_{h}(\boldsymbol{u}, p)$ & rate \\
\hline $1 / 8$ & $9.691 \times 10^{-3}$ & - & $1.672 \times 10^{-1}$ & - \\
$1 / 16$ & $2.540 \times 10^{-3}$ & 1.93 & $5.771 \times 10^{-2}$ & 1.53 \\
$1 / 32$ & $6.446 \times 10^{-4}$ & 1.97 & $2.127 \times 10^{-2}$ & 1.43 \\
$1 / 64$ & $1.604 \times 10^{-4}$ & 2.00 & $8.119 \times 10^{-2}$ & 1.38 \\
$1 / 128$ & $3.815 \times 10^{-5}$ & 2.07 & $3.139 \times 10^{-3}$ & 1.37 \\
\hline
\end{tabular}

Table 6: Convergence rates for the Marchuk-Yanenko scheme (Algorithm 2) with $k=1 / 100 ; r=r^{*}, \kappa=0.1$ and $g=0.5$. 


\begin{tabular}{|c|cccc|}
\hline$h$ & $e_{h}(\boldsymbol{u})$ & rate & $e_{h}(\boldsymbol{u}, p)$ & rate \\
\hline $1 / 8$ & $9.093 \times 10^{-3}$ & - & $1.867 \times 10^{-1}$ & - \\
$1 / 16$ & $2.492 \times 10^{-3}$ & 1.86 & $7.132 \times 10^{-2}$ & 1.38 \\
$1 / 32$ & $6.331 \times 10^{-4}$ & 1.97 & $2.483 \times 10^{-2}$ & 1.52 \\
$1 / 64$ & $1.552 \times 10^{-4}$ & 2.02 & $8.914 \times 10^{-2}$ & 1.47 \\
$1 / 128$ & $3.660 \times 10^{-5}$ & 2.08 & $3.231 \times 10^{-3}$ & 1.46 \\
\hline
\end{tabular}

Table 7: Convergence rates for the Marchuk-Yanenko scheme (Algorithm 2) with $k=1 / 100, r=r^{*}, \kappa=0.1$ and $g=1$.

\begin{tabular}{|c|cccc|}
\hline$h$ & $e_{h}(\boldsymbol{u})$ & rate & $e_{h}(\boldsymbol{u}, p)$ & rate \\
\hline $1 / 8$ & $9.371 \times 10^{-3}$ & - & $1.928 \times 10^{-1}$ & - \\
$1 / 16$ & $2.514 \times 10^{-3}$ & 1.89 & $7.198 \times 10^{-2}$ & 1.42 \\
$1 / 32$ & $6.292 \times 10^{-4}$ & 1.99 & $2.495 \times 10^{-2}$ & 1.52 \\
$1 / 64$ & $1.540 \times 10^{-4}$ & 2.03 & $8.893 \times 10^{-2}$ & 1.48 \\
$1 / 128$ & $3.642 \times 10^{-5}$ & 2.08 & $3.199 \times 10^{-3}$ & 1.47 \\
\hline
\end{tabular}

Table 8: Convergence rates for the Marchuk-Yanenko scheme (Algorithm 2) with $k=1 / 100 ; r=r^{*}, \kappa=0.1$ and $g=4$.

\subsection{D wall-driven driven square cavity}

We now study the behavior Algorithm 2 in presence of nonlinear effects associated with advection. We consider the unit square $\Omega=(0,1)^{2}$ with $\Gamma=(0,1) \times\{1\}$ and $S=\partial \Omega \backslash \Gamma$. We take $\boldsymbol{u}=(1,0)$ on $\Gamma$ and $\boldsymbol{f}=0$. The space-time discretization is obtained with $h=1 / 64$ and $k=5 \times 10^{-4}$. We adopt the same strategy as in [21] concerning the initial conditions: we set $\boldsymbol{u}_{0}=0$ for $\nu=1$ and then for other values of $\nu$ we take as $\boldsymbol{u}_{0}$ the steadystate solution obtained at the previous $\nu$ in the sequence $\{1,0.1,0.01\}$. We summarize in Table 9 the performances of Algorithm 2 in terms of the number of iterations required to reach the steady-state solution and the computational (CPU) time. In Figure 8, we plot the streamlines contours of the steady states reached. One can notice that for $\nu=10^{-1}$ and $\nu=10^{-2}$ the friction effects disappear.

\begin{tabular}{|c|ccc|}
\hline & $\nu=1$ & $\nu=10^{-1}$ & $\nu=10^{-2}$ \\
\hline Iter & 481 & 12934 & 317828 \\
CPU Time (Sec.) & 78.714 & 1306.783 & 31753.892 \\
\hline
\end{tabular}

Table 9: Number of iterations required for a stationary solution for the Navier-Stokes flow in 2D lid-driven cavity with $h=1 / 64, k=5 \cdot 10^{-4}$ and $g=1$. 


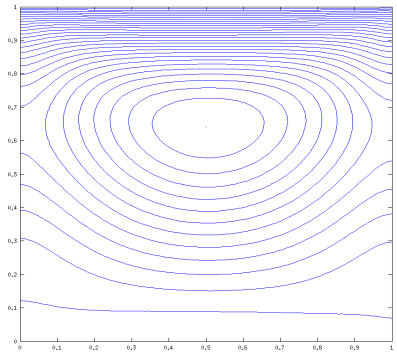

$\nu=1$

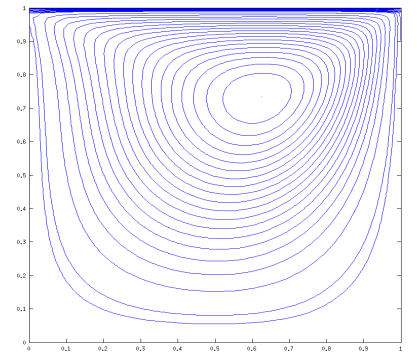

$\nu=10^{-1}$

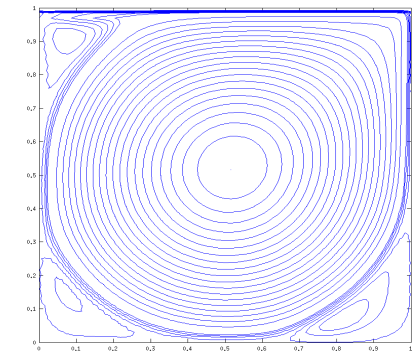

$\nu=10^{-2}$

Figure 8: Streamlines of the steady states reached for different viscosity coefficients with $h=1 / 64, k=5 \times 10^{-4}, g=1$ and $\kappa=0.1$.

\subsection{D wall-driven semi-circular cavity}

We now investigate the ability of Algorithm 2 at handling flow regions with corners and curved boundaries. We then consider a semi-circular cavity defined by

$\Omega=\left\{x \in \mathbb{R}^{2} \mid x_{2}<0\right.$ and $\left.x_{1}^{2}+x_{2}^{2}<1 / 4\right\}, \Gamma=(-1 / 2,1 / 2) \times\{0\}, S=\partial \Omega \backslash \Gamma$.

We take $\boldsymbol{u}=(1,0)$ on $\Gamma, \boldsymbol{f}=0$, and on $S$ we assume a slip boundary condition with $g=1$ and $\kappa=0.1$. We use a non uniform mesh with 4735 nodes and 9136 triangles generated by kmg2d mesh generator [35], Figure 9 . We take $k=5 \times 10^{-4}$ for $\nu=1$ and $\nu=0.1$, and $k=10^{-4}$ for $\nu<0.05$ and $\nu=0.01$. We adopt the same strategy as [21], used in Section 6.3, for the initial conditions. The penalty parameter in Algorithm 1 is $r=30$.

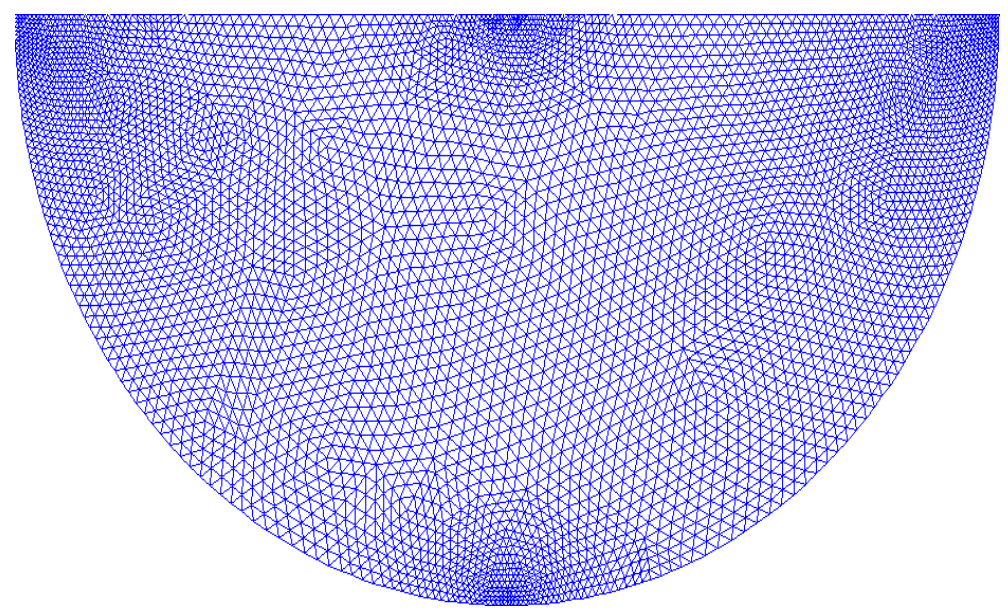

Figure 9: Triangulation on $\Omega$ with 4735 nodes and 9136 triangles

We report in Table 10 the number of iterations and CPU time (in Seconds) required to reach a stationary solution. For $\nu=0.01$, without the 

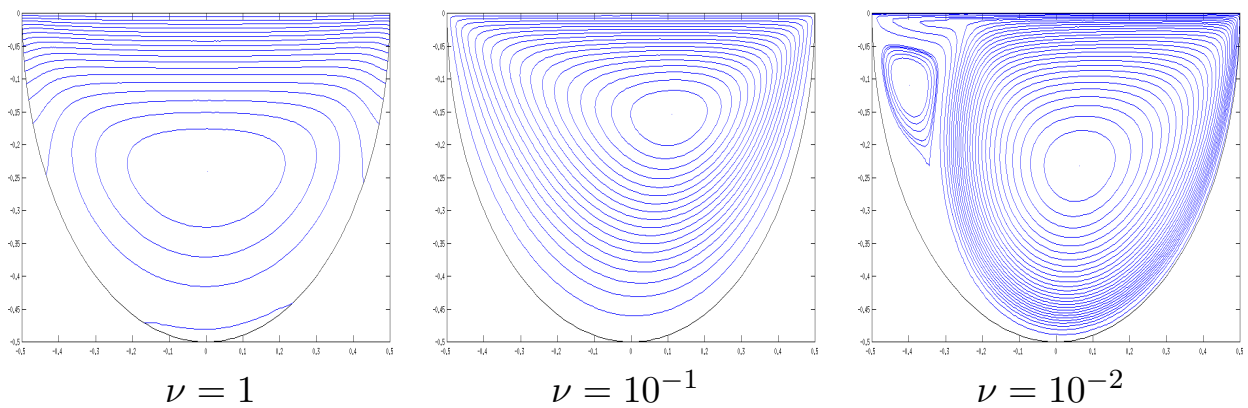

Figure 10: Streamlines of the steady states reached for different viscosity coefficients in 2D lid-driven semi-circular cavity.

intermediate value $\nu=0.05$, the solution does not reach a steady state but the $L^{2}$-error oscillates around $10^{-5}$. As shown in Figure 10, the friction effects disappear for $\nu<1$.

\begin{tabular}{|c|cccc|}
\hline & $\nu=1$ & $\nu=10^{-1}$ & $\nu=5 \times 10^{-2}$ & $\nu=10^{-2}$ \\
\hline Iter & 141 & 4984 & 13856 & 168261 \\
CPU Time (Sec.) & 205.121 & 878.624 & 1860.471 & 17852.658 \\
\hline
\end{tabular}

Table 10: Number of iterations required for a stationary solution for the Navier-Stokes flow in 2D lid-driven semi-circular cavity.

\subsection{D wall-driven cavity}

We consider the cubic cavity $\Omega=(0,1)^{3}$ with $\nu=1$ and assume a moving wall at $\Gamma=\{z=1\}$, i.e. $\boldsymbol{u}=(1,0,0)$. On the remaining part of the boundary $S=\partial \Omega \backslash \Gamma$, we assume the slip boundary condition with $\kappa=0.1$.

Applying the same procedure on a coarse mesh, as in the previous section, we find that $r^{*} \approx 25$. Figure 11 shows the velocity field at the boundary for $g=1$ and the magnitude of tangential velocity $\left\|\boldsymbol{u}_{\boldsymbol{\tau}}\right\|$. Numerical experiments also show that, for the 3D lid-driven cavity, the friction always occurs (i.e., $\left\|\boldsymbol{u}_{\boldsymbol{\tau}}\right\| \rightarrow\left\|\boldsymbol{u}_{\boldsymbol{\tau}}^{*}\right\| \neq 0$ as $g \rightarrow+\infty$ ). Table 11 summarizes the performances of Algorithm 1. We can notice that, for the 3D lid driven cavity Stokes flow, the proposed method is virtually independent of the mesh size. Note that the CPU times given include the time for assembling the matrices and performing the incomplete LU factorization.

We report in Table 12 the behavior of the Marchuk-Yanenko scheme (Algorithm 2) with $\nu=1, \kappa=0.1, k=10^{-3}$ and various values of $g$ and $h$. Note that the advection matrix in (4.7) is assembled each time step, leading to significant additional computational time. For this reason, we limit the mesh size to $h=1 / 20$ (instead of $h=1 / 32$ as in the Stokes case) 


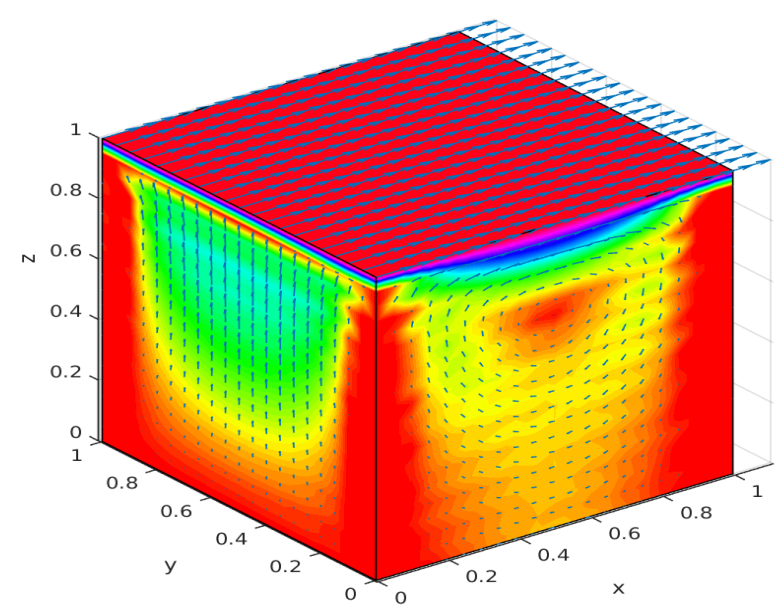

Figure 11: Velocity field and $\left\|\boldsymbol{u}_{\boldsymbol{\tau}}\right\|$ on the boundary for the Stokes problem with $\nu=1 g=1, \kappa=0.1, h=1 / 20$ and $k=10^{-3}$.

\begin{tabular}{|c|cc|cc|cc|}
\hline Mesh size $h$ & \multicolumn{2}{|c|}{$g=0.5$} & \multicolumn{2}{c|}{$g=1$} & \multicolumn{2}{c|}{$g=4$} \\
\hline & Iter & CPU (sec.) & Iter & CPU (sec.) & Iter & CPU (sec.) c \\
\hline $1 / 4$ & 40 & 0.638 & 37 & 0.201 & 75 & 0.264 \\
$1 / 8$ & 55 & 1.280 & 38 & 0.817 & 65 & 1.246 \\
$1 / 16$ & 53 & 36.313 & 43 & 37.176 & 52 & 36.867 \\
$1 / 32$ & 56 & 2212.349 & 39 & 2039.271 & 50 & 2144.049 \\
\hline
\end{tabular}

Table 11: Performances of Algorithm 1 with $r=r^{*}=25$ and $\kappa=.1$ for the 3D lid driven cavity.

\begin{tabular}{|c|cc|cc|cc|}
\hline Mesh size $h$ & \multicolumn{2}{|c|}{$g=0.5$} & \multicolumn{2}{c|}{$g=1$} & \multicolumn{2}{c|}{$g=4$} \\
\hline & Iter & CPU (sec.) & Iter & CPU (sec.) & Iter & CPU (sec.) c \\
\hline $1 / 4$ & 149 & 2.532 & 103 & 2.328 & 77 & 2.067 \\
$1 / 8$ & 244 & 32.241 & 217 & 23.785 & 188 & 19.391 \\
$1 / 16$ & 301 & 582.530 & 295 & 479.717 & 286 & 388.726 \\
$1 / 20$ & 312 & 2429.153 & 311 & 2371.602 & 300 & 1922.832 \\
\hline
\end{tabular}

Table 12: Number of iterations required for a stationary solution and CPU times (in seconds) for the Navier-Stokes flow in 3D lid-driven cavity, $k=$ $10^{-3}$. 
To study the nonlinear effects associated with advection, we vary the viscosity coefficient $\nu$ from 1 to $10^{-2}$, while keeping fixed $g=1$ and $\kappa=0.1$. For the discretization, we take $h=1 / 20$ and $k=0.0005$. For the initial conditions, we use the strategy described in Section 6.3 ([21]). We report in Table 13 the number of iterations required for a steady-state solution and the CPU time (in Seconds). As one can see in Figure 12, the friction always occurs for all values of the viscosity.

\begin{tabular}{|c|ccc|}
\hline & $\nu=1$ & $\nu=10^{-1}$ & $\nu=10^{-2}$ \\
\hline Iter & 562 & 2185 & 17389 \\
CPU Time (Sec.) & 4285.250 & 16267.625 & 133824.981 \\
\hline
\end{tabular}

Table 13: Number of iterations required for a stationary solution for the Navier-Stokes flow in 3D lid-driven cavity with $h=1 / 20, k=5 \cdot 10^{-4}$ and $g=1$.

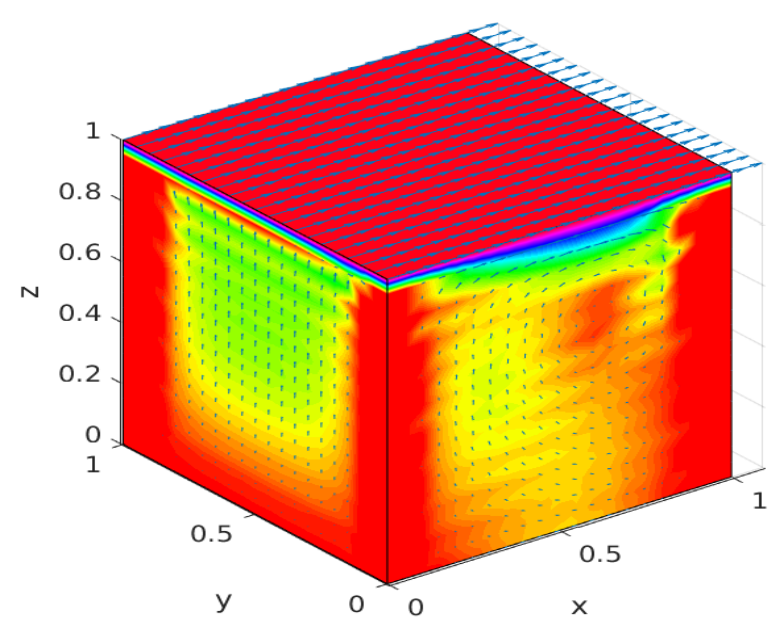

Figure 12: Velocity field and $\left\|\boldsymbol{u}_{\boldsymbol{\tau}}\right\|$ on the boundary for the Navier-Stokes flow with $\nu=10^{-2}, g=1, \kappa=0.1, h=1 / 20$ and $k=5 \times 10^{-4}$.

\section{Conclusion}

We have studied some new numerical methods for the Stokes/Navier-Stokes flow driven by nonlinear slip boundary conditions. The main advantage of the proposed methods, for the Stokes problem, is that the matrices involved are constant during the iterative process. Then, a complete or incomplete factorization can be carried out once and for all in the initialization step. Numerical experiments (in 2D and 3D) show that our methods are scalable, 
i.e. the number of iterations required for convergence is virtually independent of mesh size. Numerical experiments with different viscosity coefficient show that the proposed method can handle nonlinear effects associated with advection, if certain precautions are taken concerning the initial conditions and the time step.

Acknowledgment The authors would like to thank the referees for their constructive remarks. The first author has benefited from the financial support of National Research Foundation of South Africa.

\section{References}

[1] R. An, Y. Li, and K. Li. Solvability of Navier-Stokes equations with leak boundary conditions. Acta Math. Appl. Sin. Engl. Ser., 25:225$234,2009$.

[2] M. Ayadi, L. Baffico, M.K. Gdoura, and T. Sassi. Error estimates for Stokes problem with Tresca friction conditions. ESAIM Math. Model. Numer. Anal., 48:1413-1429, 2014.

[3] M. Ayadi, M.K. Gdoura, and T. Sassi. Mixed formulation for Stokes problem with Tresca friction. C. R. Acad. Sci. Paris, Ser. I, 348:1069$1072,2010$.

[4] P. Ciarlet. Finite Element Method for Elliptic Problems. North Holland, Amsterdam, 1978.

[5] E.J. Dean and R. Glowinski. On the numerical solution of the elliptic Monge-Ampère equation in dimension two: A least squares approach. partial differential equations. Partial Differential Equations, volume 16 of Computational Methods in Applied Sciences, pages 43-63. Springer, Dordrecht, 2008.

[6] E.J. Dean, R. Glowinski, and G. Guidoboni. On the numerical simulation of Bingham visco-plastic flow: Old and new results. J. NonNewtonian fluid Mech., 142(1-3):36-62, 2007.

[7] J.K. Djoko and M. Mbehou. Finite element analysis for Stokes and Navier Stokes equations driven by threshold slip boundary conditions. Int. J. Numer. Anal. Model. Ser. B, 4:235-255, 2013.

[8] G. Duvaut and J.-L. Lions. Inequalities in Mechanics and Physics, volume 219 of Grundlehren der Mathematischen Wissenschaften. SpringerVerlag, Berlin, 1976.

[9] I. Ekeland and R. Temam. Convex Analysis and Aariational Problems. SIAM, Philadelphia, 2nd edition, 1999. 
[10] M. Fortin and R. Glowinski. Augmented Lagrangian Methods: Applications to the Numerical Solution of Boundary Value Problems. NorthHolland, Amsterdam, 1983.

[11] H. Fujita. A mathematical analysis of motions of viscous incompressible fluid under leak or slip boundary conditions. RIMS Kōkyūroko, 888:199-216, 1994.

[12] H. Fujita. Non-stationary Stokes flows under leak boundary conditions of friction type. J. Comput. Appl. Math., 19:1-8, 2001.

[13] H. Fujita. A coherent analysis of Stokes flows under boundary conditions of friction type. J. Comput. Appl. Math., 149:57-69, 2002.

[14] H. Fujita. Variational inequalities and nonlinear semi-groups applied to certain nonlinear problems for the Stokes equation. In Tosio Kato's Method and Principle for Evolution Equations in Mathematical Physics, Saporo, July 27-29, 2001, RIMS Kyoto University, pages 70-85, 2002.

[15] H. Fujita and H. Kawarada. Distribution theoretic approach to fictitious domain method for Neumann problems. East-West Journal of Numerical Mathematics, 3:111-126, 1995.

[16] H. Fujita and H. Kawarada. Variational inequalities for the Stokes equation with boundary conditions of friction type. In Recent Developments in Domain Decomposition Methods and Flow Problems, Kyoto, 1996, volume 11 of GAKUTO International Series Mathematical Sciencies and Applications, pages 15-33, 1998.

[17] H. Fujita, H. Kawarada, and A. Sasamoto. Analytical and numerical approaches to stationary flow problems with leak and slip boundary conditions. In Proceedings of 2nd Japan-China Seminar on Numerical Mathematics, volume 14 of Kinokuniya LN in Numerical and Applied Analysis, pages 17-31, 1995.

[18] M.K. Gdoura, J. Koko, and T. Sasi. Domain decomposition method for Stokes problem with Tresca friction. In Domain decomposition methods in science and engineering XX, volume 91 of Lect. Notes Comput. Sci. Eng., pages 655-662. Springer, Heidelberg, 2013.

[19] V. Girault and P.A. Raviart. Finite Element Methods for Navier-Stokes Equations: Theory and Algorithms. Springer-Verlag, 1986.

[20] R. Glowinski. Numerical Methods for Nonlinear Variational Problems. Computational Physics. Springer, Berlin, Heidelberg, 2008.

[21] R. Glowinski, G. Guidoboni, and T.-W. Pan. Wall-driven incompressible viscous flow in a two-diemnsional semi-circular cavity. J. comput. Physics, 216:76-91, 2006. 
[22] R. Glowinski, J. L. Lions, and R. Trémolières. Numerical Analysis of Variational Inequalities, volume 8 of Studies in Mathematics and its Applications. North-Holland, Amsterdam, 1981.

[23] R. Glowinski and P. Le Tallec P. Augmented Lagrangian and operatorsplitting Methods in Nonlinear Mechanics. Studies in Applied Mathematics. SIAM, Philadelphia, 1989.

[24] R. Glowinski, T.W. Pan, and J. Périaux. Distributed Lagrange multiplier methods for incompressible viscous flow around moving rigid bodies. Comput. Methods. Appl. Mech. Engrg., 151:181-194, 1998.

[25] R. Glowinski. Finite element methods for incompressible viscous flow. In Handbook of Numerical Analysis, P.G Ciarlet and J.L Lions, eds, vol. IX, North Holland; Amsterdam, 3-1176, 2003.

[26] R. Glowinski, and A. Wachs. On the Numerical simulation of Bingham visco-plastic flow. In Handbook of Numerical Analysis, P.G Ciarlet, R. Glowinski and J. Xu, eds, vol. XVI, North Holland; Amsterdam, 483-717, 2011.

[27] W. Han and B.D. Reddy. Plasticity: Mathematical Theory and Numerical Analysis, volume 9 of Interdisciplinary Applied Mathematics. Springer, New York, 2nd edition, 2013.

[28] W. Han, M. Shillor, and M. Sofonea. Analysis and Approximation of Contact Problems with Adhesion or Damage, volume 276 of Pure and Applied Mathematics. Chapman and Hall/CRC, Boca Raton, FL, 2006.

[29] T. Hughes, L. Franca, and M. Balestra. A new finite element formulation for CFD: V. Circumventing the Babuska-Brezzi condition: A stable Petrov-Galerkin formulation of the Stokes problem accommodating equal-order interpolations. Comput. Methods. Appl. Mech. Engrg., 59:85-99, 1986.

[30] T. Kashiwabara. On a finite element approximation of the Stokes equations under a slip boundary condition of the friction type. J. Indust. Appl. Math, 30:227-261, 2013.

[31] T. Kashiwabara. On a strong solution of the non stationary NavierStokes equations under slip or leak boundary conditions. J. Differential Equations, 254:756-778, 2013.

[32] J. Koko. Lagrangian multiplier based domain decomposition methods for a nonlinear sedimentary basin problem. Comput. Geosci., 11:307317, 2007. 
[33] J. Koko. Uzawa block relaxation for the unilateral contact problem. J. Comput. Appl. Math., 235:2343-2356, 2011.

[34] J. Koko and S. Jehan-Beson. An augmented Lagrangian method for $T V_{g}+L^{1}$-norm minimization. J. Math. Imaging Vis., 38:182-196, 2010.

[35] J. Koko. A MATLAB mesh generator for two-dimensional finite element methd. Appl. Math. Comput., 250:650-664, 2015.

[36] N. Kikuchi and J.T. Oden. Contact Problems in Elasticity: A Study of Variational Inequality and Finite Element Methods, SIAM, Philadelphia, PA, 1988.

[37] R. Kucera, J. Haslinger, V. Satek, and M. Jarosova. Efficient methods for solving the Stokes problem with slip boundary conditions. Submited.

[38] R. Kucera, V. Satek, M. Jarosova, and T. Kozubek. The Stokes flow with friction. In Proceeding of the 12th Int. Confrence of Numerical Analysis and Applied Mathematics, Rhodes, GR, 2014. To appear.

[39] Y. Li and R. An. Penalty finite element method for Navier-Stokes equations with nonlinear slip boundary conditions. Int. J. Numer. Methods Fluids, 69:550-566, 2011.

[40] Y. Li and K. Li. Penalty finite element method for Stokes problem with nonlinear slip boundary conditions. Appl. Math. Computations, 204:216-226, 2008.

[41] R. Rannacher. Finite element methods for the incompressible NavierStokes equations. In J. G. Heywood G. Galdi and R. Rannacher, editors, Fundamenta Directions in Mathematical Fluid Mechanics, pages 191293. Springer, 2000.

[42] I.J. Rao and K.R. Rajagopal. The effect of the slip boundary condition on the flow of fluids in a channel. Acta Mech., 135:113-126, 1999.

[43] C. Le Roux. Steady Stokes flows with threshold slip boundary conditions. Math. Models Methods Appl. Sci., 15:1141-1168, 2005.

[44] C. Le Roux and A. Tani. Steady solutions of the Navier-Stokes equations with threshold slip boundary conditions. Math. Methods Appl. Sci., 30:595-624, 2007.

[45] F. Saidi. Non-Newtonian Stokes flow with frictional boundary conditions. Math. Model. and Anal., 12:483-495, 2007.

[46] N. Saito. On the Stokes equations with the leak and slip boundary conditions of friction type: regularity of solutions. RIMS Kōkyūroko, 40:345-383, 2004. 
[47] M. Shillor, M. Sofonea, and J.J. Telega. Models and Analysis of Quasistatic Contact: Variational Methods, volume 655 of Lectures Notes in Physics. Springer, Berlin, Heidelberg, 2004.

[48] R. Temam. Navier Stokes equations: Theory and Numerical Analysis. AMS Chelsea publishing, 2001. 